\title{
Review Article \\ Review of Herbal Traditional Chinese Medicine for the Treatment of Diabetic Nephropathy
}

\author{
Guang-dong Sun, Chao-yuan Li, Wen-peng Cui, Qiao-yan Guo, Chang-qing Dong, \\ Hong-bin Zou, Shu-jun Liu, Wen-peng Dong, and Li-ning Miao \\ Department of Nephrology, Second Hospital of Jilin University, Changchun 130041, China \\ Correspondence should be addressed to Guang-dong Sun; sungd@jlu.edu.cn and Li-ning Miao; miaolining55@163.com
}

Received 28 May 2015; Accepted 22 July 2015

Academic Editor: David W. Powell

Copyright (c) 2016 Guang-dong Sun et al. This is an open access article distributed under the Creative Commons Attribution License, which permits unrestricted use, distribution, and reproduction in any medium, provided the original work is properly cited.

\begin{abstract}
Diabetic nephropathy (DN) is the most serious chronic complications of diabetes; $20-40 \%$ of diabetic patients develop into end stage renal disease (ESRD). However, exact pathogenesis of DN is not fully clear and we have great difficulties in curing DN; poor treatment of $\mathrm{DN}$ led to high chances of mortality worldwide. A lot of western medicines such as ACEI and ARB have been demonstrated to protect renal function of DN but are not enough to delay or retard the progression of DN; therefore, exploring exact and feasible drug is current research hotspot in medicine. Traditional Chinese medicine (TCM) has been widely used to treat and control diabetes and its complications such as $\mathrm{DN}$ in a lot of scientific researches, which will give insights into the mechanism of DN, but they are not enough to reveal all the details. In this paper, we summarize the applications of herbal TCM preparations, single herbal TCM, and/or monomers from herbal TCM in the treatment of DN in the recent 10 years, depicting the renal protective effects and the corresponding mechanism, through which we shed light on the renal protective roles of TCM in DN with a particular focus on the molecular basis of the effect and provide a beneficial supplement to the drug therapy for DN.
\end{abstract}

\section{Introduction}

Diabetic nephropathy (DN) is a widely recognized microvascular complication of diabetes and almost the leading cause of end-stage kidney failure worldwide responsible for morbidity and mortality [1]. Clinical manifestations of DN include initial increase in glomerular filtration (GFR), proteinuria, increased creatinine levels, and eventually decreased GFR [24]. Major pathological changes of DN are virtually indistinguishable in both type 1 and type 2 diabetes, including mesangial expansion, extracellular matrix (ECM) accumulations, tubulointerstitial fibrosis, and glomerular sclerosis. Hyaline arteriolosclerosis is often prominent in the established DN pathological features caused by endothelial dysfunction and inflammation [5-7].

Multiple factors have been implicated in the pathogenesis of DN including hyperglycemia induced activation of advanced glycation end products (AGEs) and reactive oxygen species (ROS); JAK-STAT pathways and G protein signaling; activation of the PKC, renin-angiotensin aldosterone system
(RAAS), transforming growth factor $\beta$-Smad-mitogenactivated protein kinase (TGF- $\beta$-Smad-MAPK), deregulated expression of cyclin dependent kinases (CDK), and their inhibitors; and aberrant expression of ECM proteins, ECMdegrading enzymes, metalloproteinases, and their inhibitors [8]. The abovementioned factors can induce aberrant expression of profibrotic and proinflammatory cytokines, cell-cycle genes, and ECM genes involved in DN [9]. A large number of novel treatment options has arisen from experimental studies based on the pathogenic factors of $\mathrm{DN}$, including intensive glycemic control, precise blood pressure control, optimal RAAS blockade with ACEI/ARB, life style modifications such as exercise and dietary restrictions, and a lot of novel agents [10], but the portion of ESRD due to DN still remains high in spite of the widespread application of numerous therapeutic approaches focusing on the management of factors mentioned above [11-13]. Therefore, interventions that could effectively delay the progression of $\mathrm{DN}$ are greatly required.

In China, traditional Chinese medicine (TCM) has been widely used in the treatment of diabetes and its complications 
for a long time [14]; TCM has lots of advantages over the conventional medical approaches in the prevention of diabetic complications because of less toxicity and/or side effects [1517]. In this review, we will explore the advance of herbal TCM treatment on DN in recent 10 years, based on the experimental and clinical studies to note the scientific basis for the therapeutic effects of TCM on DN.

\section{Applications of TCM in DN}

Plants have been widely used for medical purposes long before recorded history [18]. In China, TCM emerged and influenced the surrounding countries such as Japan and South Korea; increasing popularity of TCM caused great interests in laboratory and clinical investigations in lots of diseases on its efficiency and action mechanism. TCM manifests as herbal medicine, acupuncture, moxibustion, massage, dietary therapy, and physical exercise including shadow boxing and Qigong, and herbal remedies are the focus of TCM in mainland China [19] and acupuncture is prevalent in the United States [18]. Under the urgent need for the treatment of DN, we focus on the update of the efficient herbal TCM preparations, single herbal TCM, and/or monomers from herbal TCM in DN related clinical and experimental trials, through which we explore the effective herbal TCM for DN and clearly put forward underlying mechanism in the treatment of DN.

2.1. TCM Preparations in DN. TCM preparations are applied as decoction, pill, and capsule in the treatment of DN. We will introduce the TCM preparations in alphabetical order about components of TCM preparations, therapeutic effects in clinical or experimental studies, and relevant mechanism. All the mentioned TCM preparations in this review are listed in Table 1.

2.1.1. Chaihuang Yishen Granule (CHYS). Chaihuang Yishen granule (CHYS, also called Qilong-Lishui granule) is composed of radix astragali, Dioscorea nipponica, radix bupleuri, Angelica sinensis, Pyrrosia petiolosa, Polyporus umbellatus, and Hirudo nipponica. A recent study in STZ plus uninephrectomized induced rats showed that CHYS could be a therapeutic agent for DN by blocking TGF- $\beta /$ Smad3-mediated renal fibrosis [20].

2.1.2. Compound Rhizoma Coptidis Capsule (CRCC). Compound rhizoma coptidis capsule (CRCC) is composed of rhizoma coptidis, Kudzu root, dwarf lilyturf,and Loquat leaf. CRCC has been shown to protect renal function and slow down the progression of DN by the suppression of TGF- $\beta 1$ and type IV collagen expression in STZ induced diabetic rats [21].

2.1.3. Compound Shenhua Tablet (CST). Compound Shenhua Tablet (CST), is composed of radix astragali, fructus ligustri lucidi, rhizoma zedoaria, and honeysuckle. CST treatment in STZ induced diabetic rats showed that urine mAlb, Scr, BUN, Glu, TG, and TC were significantly lower than the diabetic model group [22].
2.1.4. Danggui Buxue Tang (DBT). Danggui buxue tang (DBT), a preparation including radix astragaliandradix Angelica sinensis, has been shown to partially attenuate the increases in blood glucose, TG, and $\mathrm{CHO}$, and DBT was supposed to retard DN progression by suppressing TGF$\beta 1$ expression in STZ induced diabetic rats [23]. In the HG stimulated glomerular mesangial cells, DBT could inhibit cell proliferation and expression of LN, FN, and collagen IV indicating the renoprotective effect of DBT on DN at the early stages [24].

2.1.5. Danggui Shaoyao San (DSS). Danggui Shaoyao San (DSS) is a famous TCM formula comprising six herbal medicines: radix Paeoniae Alba, radix Angelica sinensis, rhizoma Chuanxiong, Poria cocos, rhizoma Atractylodis macrocephala, and rhizoma Alismatis. DSS has been shown to protect renal function in STZ induced diabetic rats through regulating plasma glucose and attenuating AGEs expression in diabetic glomeruli [25].

2.1.6. Fufang Xue Shuan Tong (FXST). Fufang Xue Shuan Tong (FXST) capsule is composed of radix notoginseng, Salvia miltiorrhiza, XuanShen, and radix astragali and has been used to treat DN for many years. High dose of FXST treatment could prevent glomerular hypertrophy and mesangial matrix expansion through regulation of oxidative stress including increasing SOD activities and decreasing MDA levels in the kidney of HFD-fed plus STZ induced rats [26].

2.1.7. Hachimijiogan (HJG). A most popular herbal medicine in Japanese Kampo, Hachimijiogan (HJG, Ba Wei Di Huang Wan in Chinese), is extracted from a mixture of Rehmannia radix, corni fructus, Dioscorea rhizome, Hoelen, Alismatis rhizome, Moutan cortex, Cinnamomi cortex, and Aconiti tuber. In subtotal nephrectomy plus STZ induced rats, HJG could reduce blood glucose and urinary protein excretion levels and increase Ccr; furthermore, HJG could ameliorate oxidative stress and AGEs formation associated with DN and subsequently prevent the development of renal lesions including glomerular sclerosis, tubulointerstitial lesions, mesangial expansions, and atherosclerosis [27]. In spontaneous diabetic WBN/Kob rats with DN, HJG could prevent $\mathrm{DN}$ progression through several established biomarkers in plasma [28] and by reducing renal oxidative injury and expression of $\mathrm{FN}$ and TGF- $\beta 1$ proteins [29]. In OLETF rats, HJG could reduce TGF- $\beta 1, F N$, iNOS, and COX-2 expressions in kidney cortex, urinary protein excretion was decreased, Ccr levels were improved, and serum glycosylated protein and AGEs were reduced effectively; data mentioned above suggested that HJG has beneficial effect on the DN progression [30].

2.1.8. Hu-Lu-Ba-Wan (HLBW). Hu-Lu-Ba-Wan (HLBW), composed of Trigonella foenum-graecum L. (TFG) and Psoralea corylifolia L. $(P C)$, has been shown to improve hyperglycemia, hyperlipidemia, and proteinuria in the HFD-fed plus STZ induced rats and could play renoprotective effect in attenuating renal oxidative stress via $\mathrm{PKC}-\alpha / \mathrm{NADPH}$ oxidative pathway [31]. 


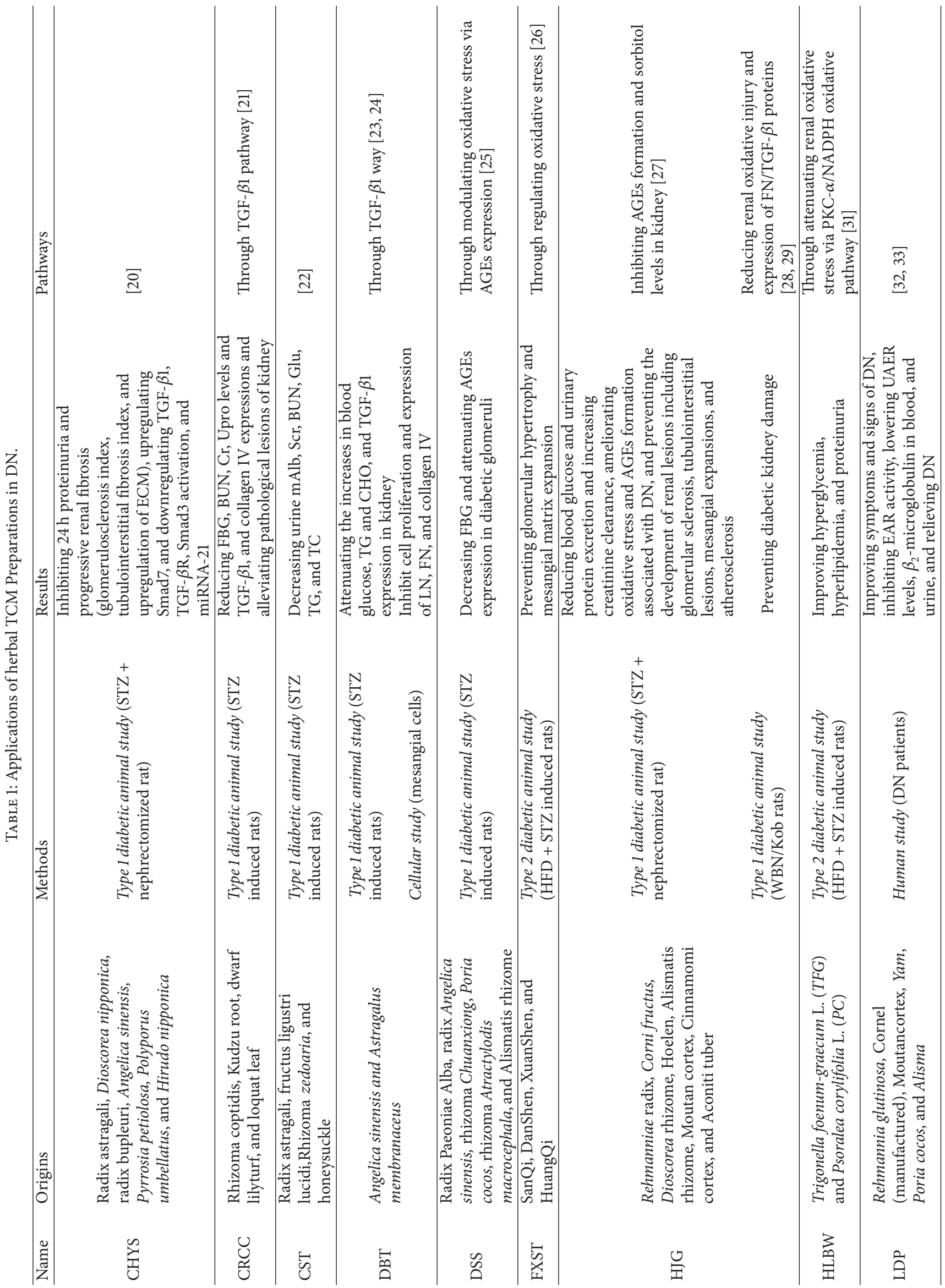




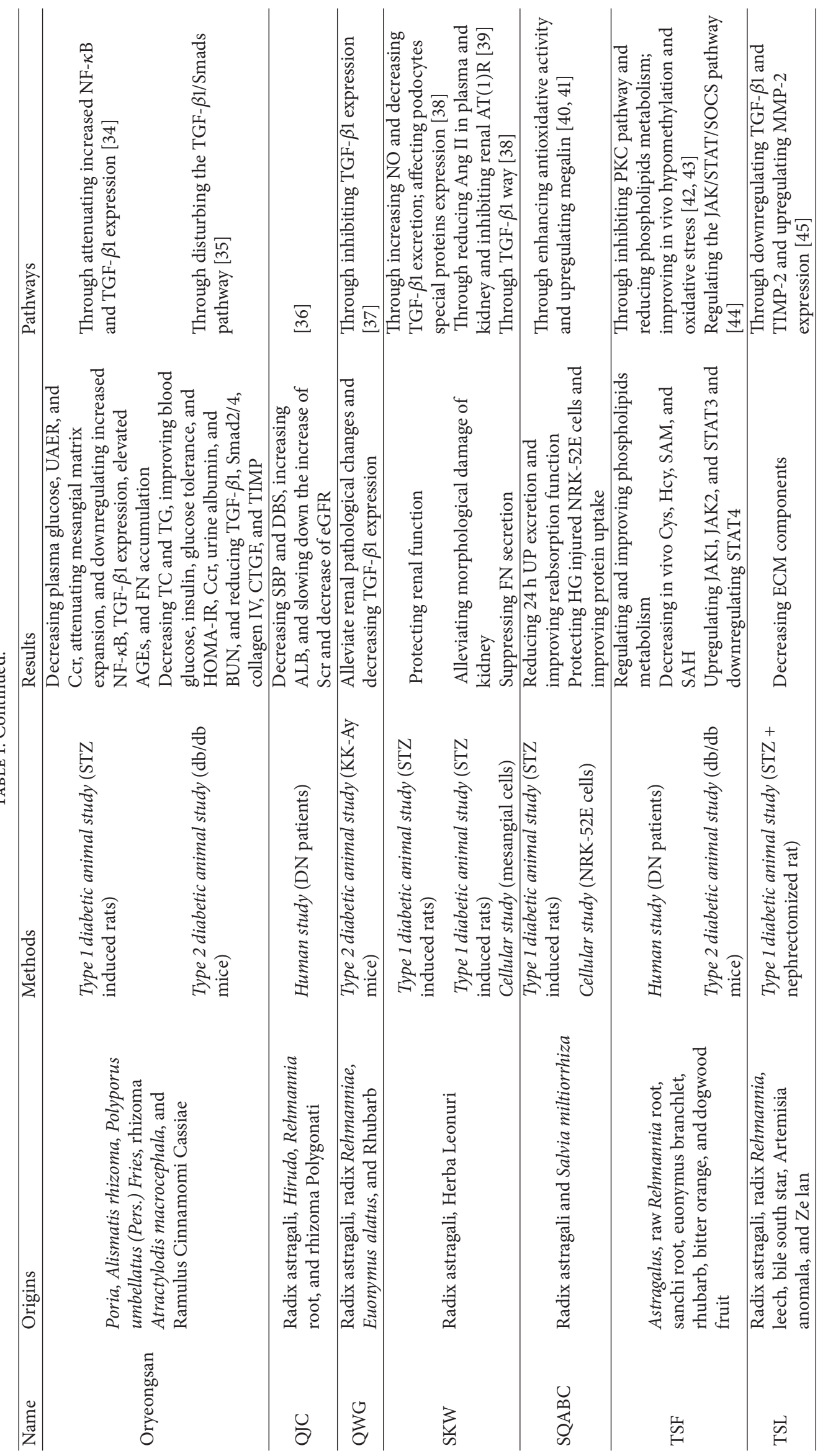




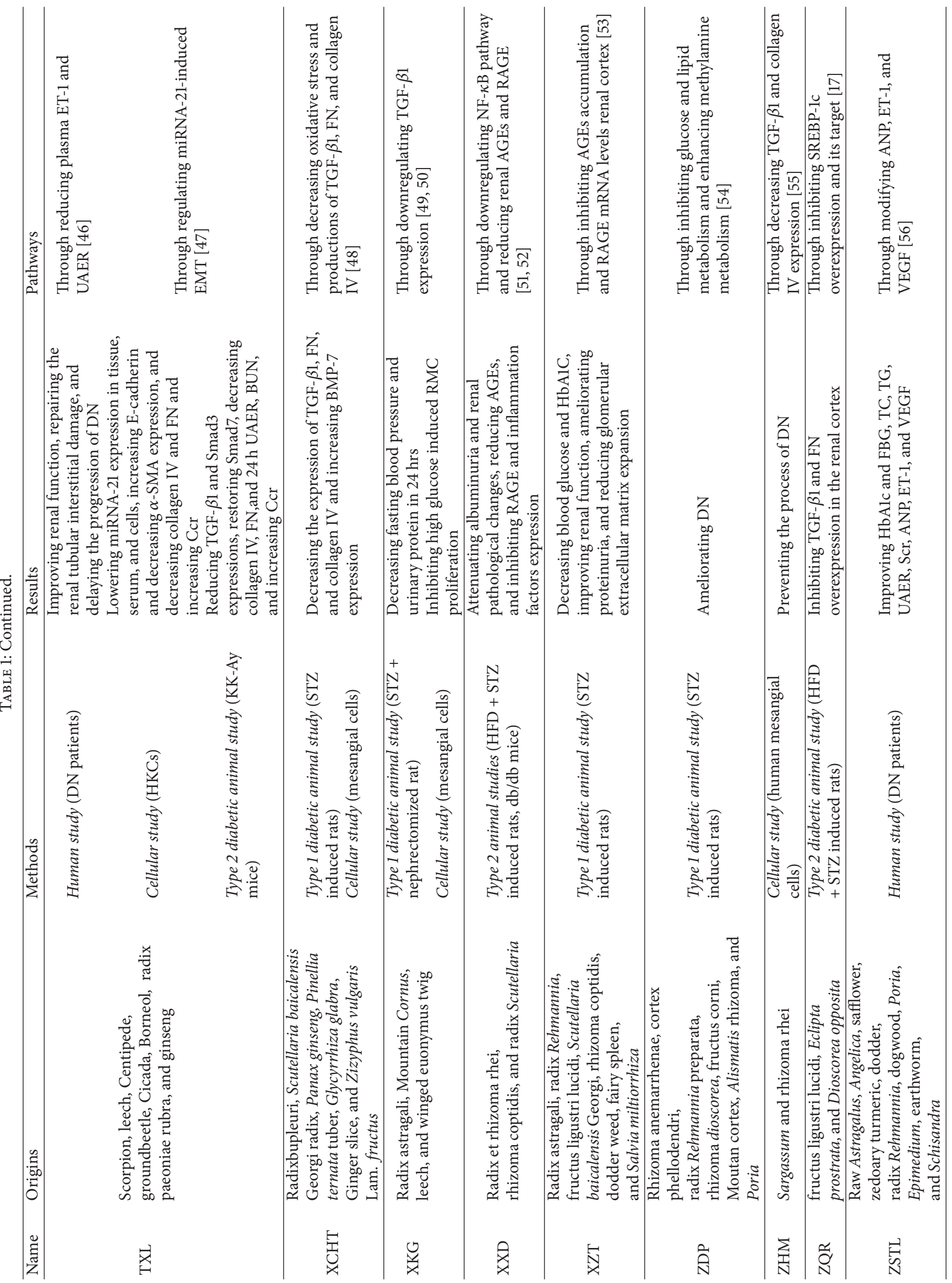


2.1.9. Liuwei Dihuang Pill (LDP). Liuwei Dihuang Pill (LDP), one formulation in the ancient Chinese medicine, includes six crude drugs: Rehmannia glutinosa, fructus corni, cortex Mountain, Dioscorea opposita, Poria cocos, and Alisma. A previous study in DN patients showed that LDP could improve symptoms and signs of DN and inhibit erythrocyte aldose reductase (EAR) activity and lower UAER levels, $\beta_{2}-$ microglobulin in blood and urine without affecting blood glucose, lipids, and mean arterial pressure [32]. LDP treatment in type 2 diabetic patients was found to be associated with the relief of DN [33]. Liuwei Dihuang (LW) decoction has also been proven to relieve early $\mathrm{DN}$ abnormalities mediated by suppression of renal entothelin-1-reactive oxidative species (ET-ROS) system and escalating MMPs activity [57], and LW without fructus corni could alleviate DN by combined suppression of ET-ROS axis with modulating hypoglycemic effects in STZ induced diabetic rats [58].

2.1.10. Oryeongsan (Wulingsan). Oryeongsan (Wulingsan), also named as Hoelen Five Herb Formula, is composed of five crude drugs: Poria, Alismatis rhizoma, Polyporus umbellatus (Pers.) Fries, rhizoma Atractylodis macrocephala, and Ramulus Cinnamomi Cassiae. A previous study showed that Oryeongsan could play renal protective roles in lowering plasma glucose and ameliorating glycation-mediated renal damage through attenuating increased NF- $\kappa$ B and TGF- $\beta 1$ expression in STZ induced diabetic rats [34]. Further study showed that Oryeongsan could ameliorate insulin resistance and $\mathrm{DN}$ in $\mathrm{db} / \mathrm{db}$ mice by disturbing the TGF- $\beta 1 /$ Smads pathway [35].

2.1.11. Qizhi Jiangtang Capsule (QJC). Qizhi Jiangtang Capsule (QJC) is composed of four crude drugs: radix astragali, Hirudo, Rehmannia root, and rhizoma Polygonati. In a multicenter randomized clinical study, QJC has been shown to reduce urinary protein effectively and delay the progression of renal function in treating $3 \mathrm{~b} \mathrm{DN}$ patients [36].

2.1.12. Qiwei Granule (QWG). Qiwei Granule (QWG) is composed of radix astragali, radix Rehmannia, Euonymus alatus, and Rhubarb. QWG could alleviate renal pathological changes and decrease TGF- $\beta 1$ expression in the type 2 diabetic KK-Ay mice, which suggested that QWG could play roles in preventing and curing DN [37].

2.1.13. Shenkangwan (SKW). Shenkangwan (SKW) is composed of two crude drugs: radix astragaliand Herba Leonuri. SKW was reported to protect renal function by increasing NO production and decreasing TGF- $\beta 1$ excretion in the mesangial cells from diabetic rats [38]; in diabetic rats SKW could reduce FN expression in kidney [59] while in rat mesangial cells SKW has been shown to suppress FN secretion via TGF- $\beta 1$ signal way [60]. Another study showed that in STZ induced diabetic rats SKW could protect renal function and alleviate the functional and structural damage of podocytes possibly by reducing desmin and increasing podocin expression [61], and SKW could offer renal protection against $\mathrm{DN}$ by reducing Ang II levels in the plasma and kidney tissues and inhibiting renal AT(1)R expressions [39]. All the data supply precise mechanism of SKW treating DN.

2.1.14. Supplementing Qi and Activating Blood Circulation $(S Q A B C)$. Supplementing Qi and activating blood circulation (SQABC) is composed of radix astragali and Salvia miltiorrhiza and has been shown to reduce $24 \mathrm{~h}$ urinary protein excretion and improve tubular reabsorption function by enhancing renal tissue activity of antioxidant and upregulating megalin expression in tubular epithelial cells in STZ induced diabetic rats [40]. Another in vitro study showed that supplementing Qi and activating blood circulation could protect HG injured NRK-52E cells and improve protein uptake by increasing megalin expression [41].

2.1.15. Tangshen Formula (TSF). Tangshen Formula (TSF) is composed of Astragalus, raw Rehmannia root, sanchi root, Euonymus branchlet, rhubarb, bitter orange, and dogwood fruit. TSF has been shown to regulate and improve phospholipids metabolism in DN patients related with inhibition of PKC pathway and the corresponding reduction of phospholipase A2 activity [42]. In a study on the Hcy metabolism of DN patients, TSF could improve in vivo hypomethylation and oxidative stress showing similar favorable effect to western medicine in the treatment of DN [43]. In the molecular mechanism study using a db/db mice model, TSF showed beneficial effects on DN treatment via regulating the JAK/STAT/SOCS signaling pathway [44].

2.1.16. Tongshenluo (TSL) Capsule. Tongshenluo (TSL) capsule is composed of six crude drugs: radix astragali, radix Rehmannia, leech, bile south star, Artemisia anomala, and Ze lan. TSL has been shown to decrease the levels of FBG, $\mathrm{HbAlc}$, and urinary mAlb in the subtotal nephrectomy plus STZ induced diabetic rats and decrease the components of ECM through downregulating TGF- $\beta 1$ and TIMP-2 and upregulating MMP-2 expression [45].

2.1.17. Tongxinluo (TXL). Tongxinluo (TXL) capsule include 8 crude drugs: scorpion, leech, centipede, ground beetle, cicada, borneol, radix paeoniae rubra, and ginseng. TXL capsule has been shown to improve renal function, repair the renal tubular interstitial damage, and delay the progression of DN patients by reducing plasma ET-1 and UAER [46]. TXL was also demonstrated to ameliorate renal function and structure by regulating miRNA-21-induced EMT, suggesting miRNA-21 may be one of the therapeutic targets for TXLC in DN [47]. Another study showed that TXL could also successfully inhibit TGF- $\beta 1$ induced EMT in DN [62].

2.1.18. Xiao Chai Hu Tang (XCHT). Xiao Chai Hu Tang (XCHT, Shosaiko-to in Japanese) is a herbal drug formula extensively applied in TCM and Japanese Kampo medicine, comprising seven medicinal plants: radix bupleuri, Scutellaria baicalensis Georgi radix, Panax ginseng, Pinellia ternata tuber, Glycyrrhiza glabra, ginger slice, and Zizyphus vulgaris Lam. fructus. XCHT has been shown to decrease the expression of TGF- $\beta 1, \mathrm{FN}$, and collagen IV accompanied with increased BMP-7 expression in STZ induced diabetic 
mice and HG stimulated RMC, which was mediated through decreasing oxidative stress and productions of TGF- $\beta 1$, FN, and collagen IV in renal cortex during the development of DN [48].

2.1.19. Xiaoke Granule (XKG). Xiaoke granule (XKG, Xiaoke Keli in Chinese) includes four crude drugs: radix astragali, Mountain Cornus, leech, and winged euonymus twig. It was reported that XKG could decrease fasting blood pressure and $24 \mathrm{~h}$ urinary protein excretion in the $3 / 4$ nephrectomy and STZ induced diabetic rats groups [49]. In the subsequent mechanism study, XKG was proved to exert renal protective effect in DN through downregulating TGF- $\beta 1$ expression in rat mesangial cells [50].

2.1.20. Xiexin Decoction (XXD). Xiexin decoction (XXD) is composed of three crude drugs including radix et rhizoma rhei, rhizoma coptidis, and radix Scutellaria and has been used for the treatment of DM for at least 1700 years. One study in HFD-fed plus STZ induced rats showed that XXD could attenuate albuminuria and renal pathological changes, reduce AGEs, inhibit RAGE and inflammation factors expression, suppress NF- $\kappa \mathrm{B}$, and downregulate renal TGF- $\beta 1$. All these data suggested that renal protective potential of XXD was involved in inhibition of inflammation through downregulating NF- $\kappa$ B pathway, reducing renal AGEs and RAGE in diabetic rats [51]. A recent study of XXD components in $\mathrm{db} / \mathrm{db}$ mice showed that multicomponent herbal therapeutic formulations could be a useful approach for the treatment of $\mathrm{DN}$ through reducing the expression of NF- $\kappa \mathrm{B}$ and TGF- $\beta 1$ [52].

2.1.21. Xianzhen Tablet (XZT). Xianzhen tablet (XZT, a Chinese patent compound recipe), is composed of astragali radix, radix Rehmannia, fructus ligustri lucidi, Scutellaria baicalensis Georgi, rhizoma coptidis, dodder weed, fairy spleen, and Salvia miltiorrhiza. XZT was reported to decrease blood glucose and HbAlc in diabetic rats, improve renal function, ameliorate proteinuria, and reduce glomerular extracellular matrix expansion and thickness of basement membrane, which was mediated by the inhibition of AGEs accumulation and RAGE mRNA levels in the kidney cortex of STZ induced diabetic rats [53].

2.1.22. Zhibai Dihuang Pill (ZDP). Zhibai Dihuang Pill (ZDP) is one of the TCM preparations, composed of rhizoma anemarrhenae, cortex phellodendri, radix Rehmannia preparata, rhizoma Dioscorea, fructus corni, cortex Moutan, rhizoma Alismatis, and Poria. ZDP has been revealed to have protective effects in experimental DN animal models and DN patients. In a recent metabonomic analysis of ZDP in the treatment of STZ induced diabetic rats, ZDP could ameliorate DN by intervening in some dominant metabolic pathways such as inhibiting glucose and lipid metabolism and enhancing methylamine metabolism [54].

2.1.23. Zao Huang Mixture (ZHM). Zao Huang Mixture (ZHM) is composed of extracts of Sargassum and rhizoma rhei. One study has shown that ZHM could prevent the process of DN by decreasing the expression of TGF- $\beta 1$ and type IV collagen in HG stimulated human glomerular mesangial cells [55].

2.1.24. Zhenqing Recipe ( $Z Q R)$. Zhenqing Recipe (ZQR), a Chinese herbal prescription composed of 3 crude drugs: fructus ligustri lucidi, Eclipta prostrate, and Dioscorea opposite, has been used to improve renal function of DN patients. In the study for the underlying mechanism, ZQR has been shown to inhibit the overexpression of TGF- $\beta 1$ and FN in the renal cortex of HFD-fed plus STZ induced diabetic rats; its renoprotective effect was mediated by inhibiting SREBP-1c overexpression and its target genes including ACC and FAS [17].

2.1.25. Zishentongluo (ZSTL). Zishentongluo (ZSTL) is composed of eleven Chinese herbs: raw Astragalus, Angelica, safflower, zedoary turmeric, Dodder, radix Rehmannia, dogwood, Poria, Epimedium, earthworm, and Schisandra. ZSTL has been shown to be superior to benazepril in improving the metabolic and renal function in DN patients at early stage partially by modifying ANP, VEGF, and ET-1 expressions [56].

2.2. Monomers/Single TCM in DN. With the development of modernization of TCM preparations and applications in the treatment of $\mathrm{DN}$, pharmacological complexity is difficult to be distinguished for the precise underlying mechanism, and, to avoid the toxicity and side effects, there is an increasing interest of single herbal TCM and/or monomers from herbal TCM in the treatment of DN, and they are more appropriate than TCM preparations to clarify the precise action mechanism on DN. All the single herbal TCM and monomers are listed in Table 2.

2.2.1. Astragalus/Radix Astragali. Astragalus (Huang Qi in Chinese), also named as radix astragali, is a TCM from Mongolian milkvetch or membranaceus milkvetch. A metaanalysis comprising 25 studies showed that Astragalus injection had more therapeutic effect in DN patients such as decreasing BUN, Scr, and urine protein and improving $\mathrm{Ccr}$ and serum albumin level [63], and rebalancing TGF- $\beta /$ Smad signaling could be a potential mechanism to prevent DN in KK-Ay mice [64]. Astragalus may protect diabetic rats kidney mediated by downregulation of Tie-2 [110], and radix astragali was reported to upregulate c-met expression in human kidney fibroblasts to delay the progression of $\mathrm{DN}$ [65]. Two major isoflavonoids in radix astragali, calycosin and calycosin-7-O-beta-D-glucoside, could inhibit HG induced mesangial cell early proliferation and AGEs-mediated cell apoptosis, suggesting these two isoflavonoids have therapeutic potential to prevent the progression of DN [111]. A recent review showed that total polysaccharides, flavonoids fractions, saponins, and several isolated compounds have antidiabetic potentials, which throw light upon further investigations that should be conducted on the treatment of DN and relevant underlying mechanism [112]. Astragaloside IV (ASI) in radix astragali is considered to be an active constituents; ASI could inhibit human tubular epithelial cells apoptosis and reduce TGF- $\beta 1$ expression, suggesting a new 


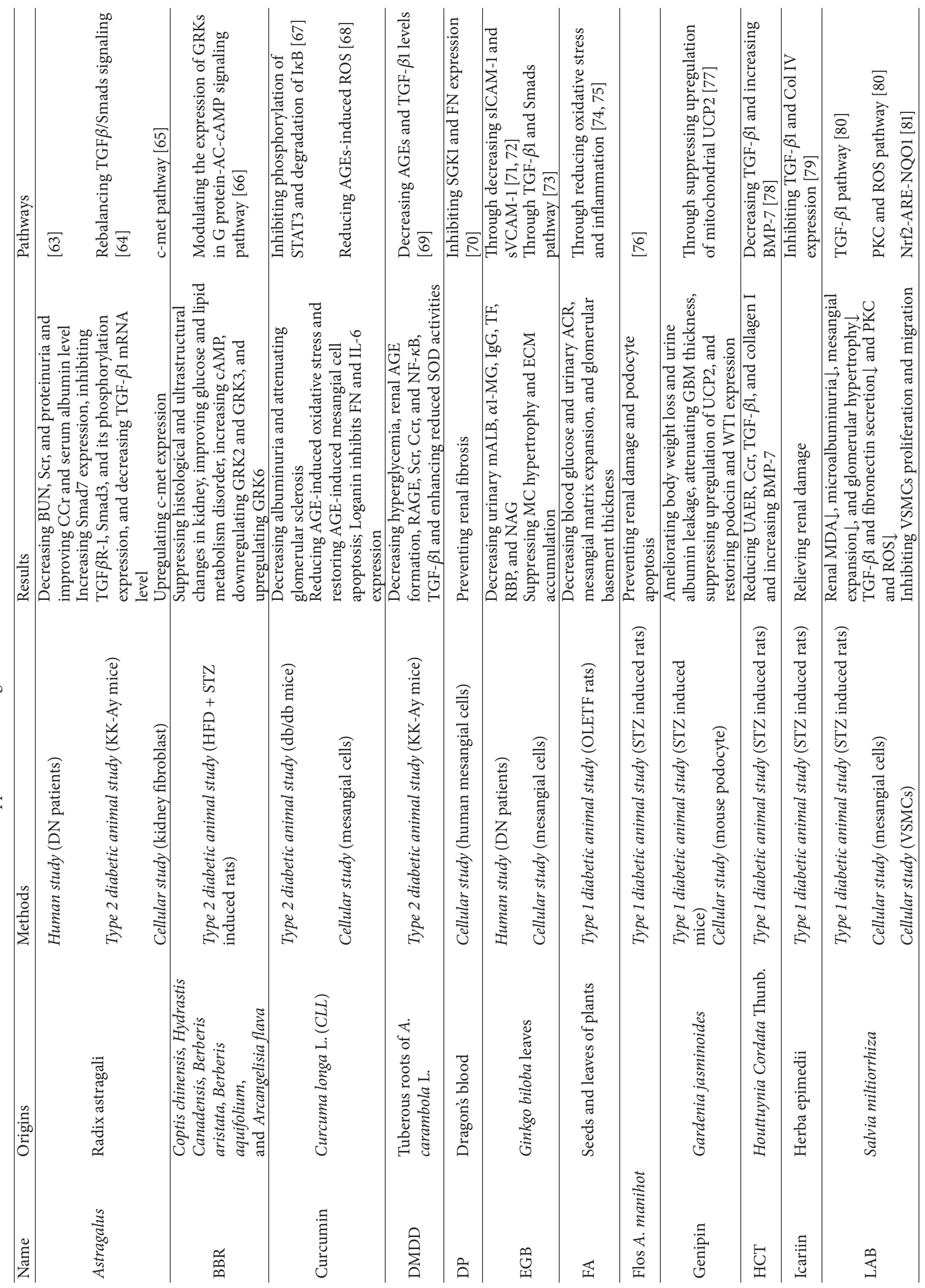




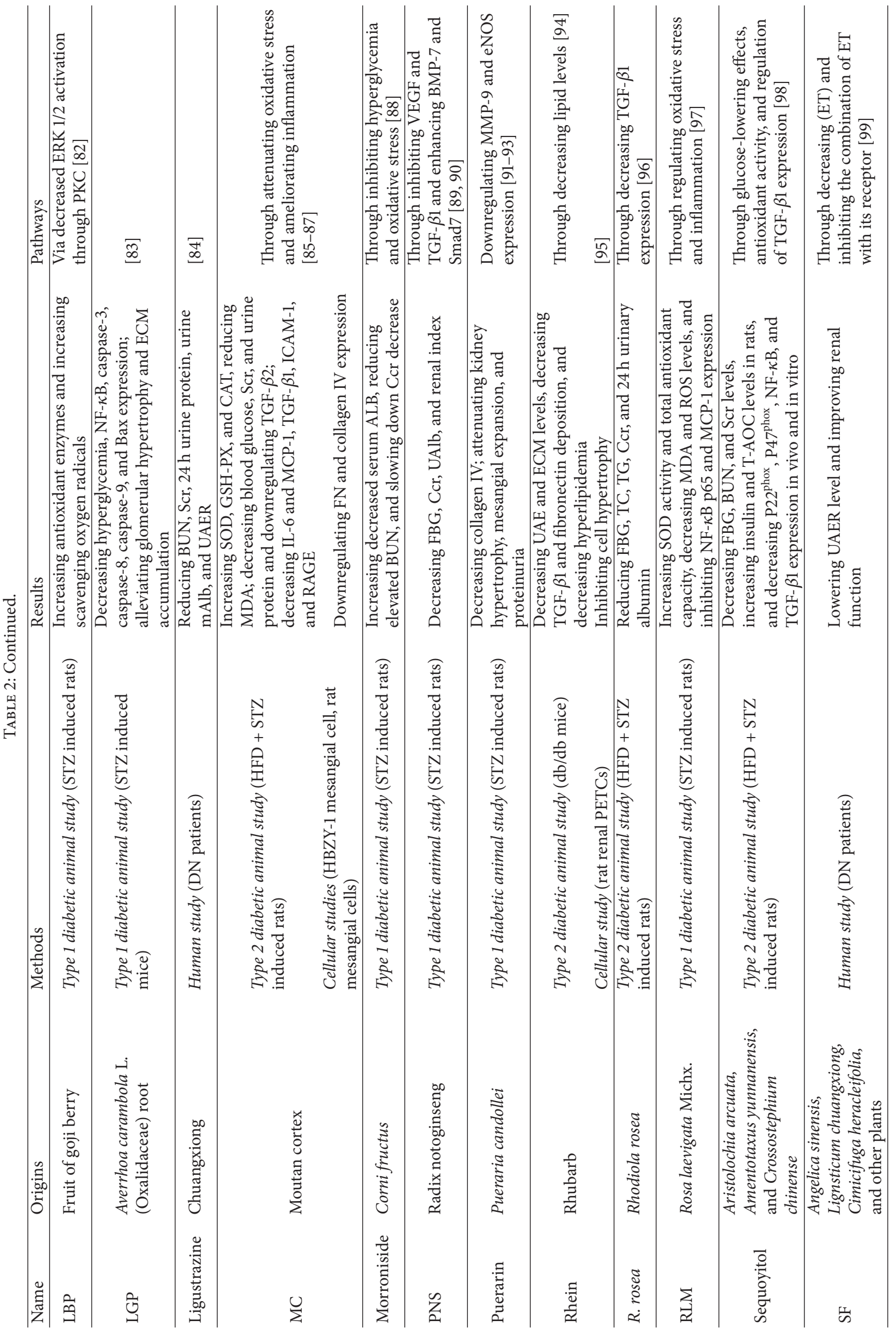




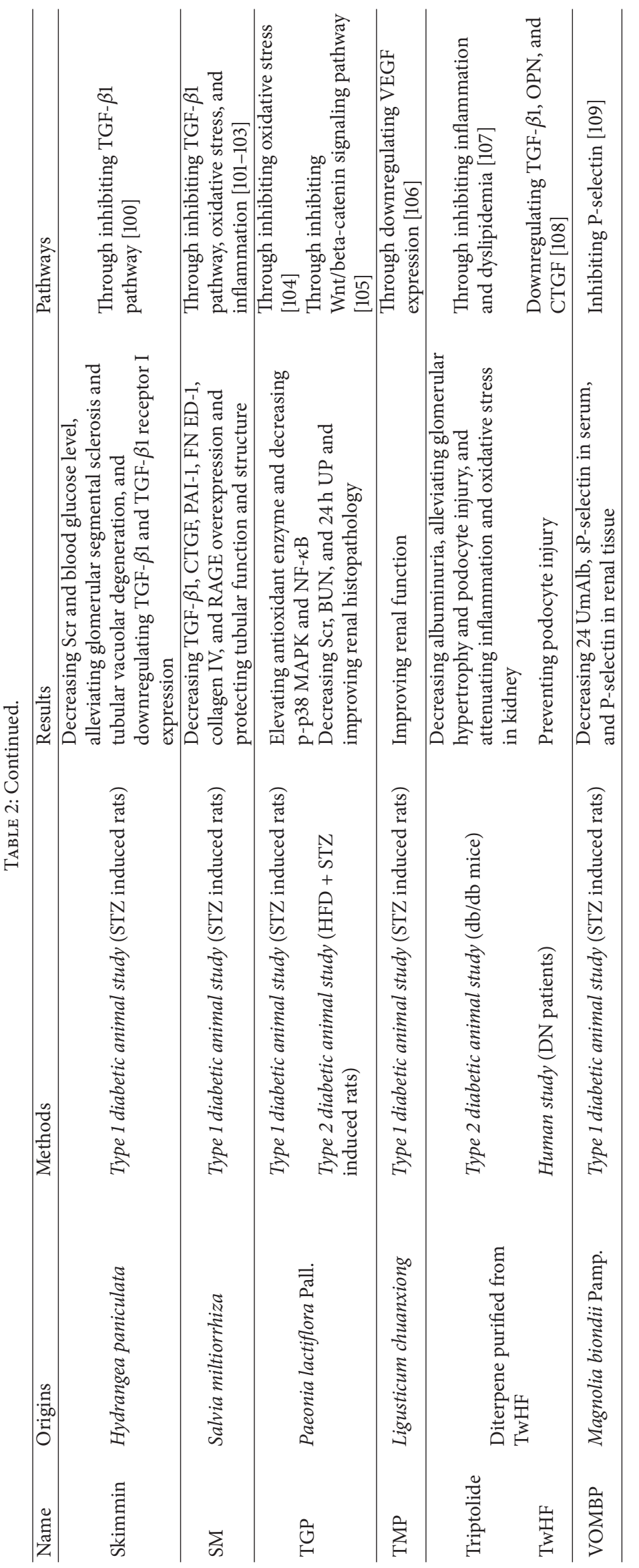


treatment for DN probably mediated by the inhibition of $\mathrm{p} 38$ MAPK pathway activation and HGF overproduction [113].

2.2.2. Berberine (BBR). Berberine (BBR), an effective compound of herbal TCM, includes Coptis chinensis, Hydrastis Canadensis, Berberis aristata, Berberis aquifolium, and Arcangelisia flava.

BBR treatment could restore renal functional parameters, improve glucose and lipid metabolism disorders, suppress alterations of histological and ultrastructural changes in kidney, and increase cAMP levels in HFD-fed plus STZ induced diabetic rats, and the renal protective effect is exerted by modulating the $\mathrm{G}$ protein-coupled receptor kinases (GRKs) in $\mathrm{G}$ protein-AC-cAMP signaling pathway [66]. A previous study showed that BBR-containing TCM could increase glucose uptake and lipid oxidation with insulin sensitivity in Zucker diabetic fatty rats [16].

2.2.3. CLL/Curcumin. Curcuma longa L. (CLL) has been widely used to prevent diabetic vascular complications in recent years. Curcumin and demethoxycurcumin are isolated from CLL and have been shown to potentially protect $\mathrm{DN}$ by reducing AGE-induced oxidative stress and restoring AGEinduced mesangial cell apoptosis [68]. In the treatment of $\mathrm{DN}$ in $\mathrm{db} / \mathrm{db}$ mice, curcumin has been shown to decrease albuminuria and attenuate glomerular sclerosis by inhibiting phosphorylation of STAT3 and degradation of I $\kappa$ B [67]. A systemic review and meta-analysis of fourteen randomized controlled trials suggested that curcumin has protective potentials on the kidneys of diabetic rats/mice [114].

\subsubsection{2-Dodecyl-6-methoxycyclohexa-2,5-diene-1,4-dione} (DMDD). 2-Dodecyl-6-methoxycyclohexa-2,5-diene-1,4dione (DMDD), isolated from the tuberous roots of $A$. carambola L. (Oxalidaceae), has been shown to enhance the reduced SOD activities in the kidney of KK-Ay mice and inhibit the progression of DN through decreasing AGEs and TGF- $\beta 1$ levels [69].

2.2.5. Dracorhodin Perchlorate (DP). Dracorhodin perchlorate (DP), one of the main compositions of Dragon's blood, has been shown to prevent and retard renal fibrosis of DN partially through inhibiting SGK1 and FN expression in human mesangial cells [70].

2.2.6. EGB. Ginkgo biloba extract (EGB), taken from the leaves of Ginkgo biloba, is a mixture containing flavonoid glycosides and has been proven to ameliorate hemodynamics, suppress PAF and ACE activities, scavenge ROS, relax vascular smooth muscles, and suppress AGEs expression. In a previous study on DN patients, EGB treatment has been shown to decrease urinary mALB, $\alpha 1-\mathrm{MG}$, IgG, TF, RBP, and NAG in DN patients compared with control group, which suggested that EGB has renoprotective effect on the early DN [71]. The subsequent mechanism study showed that EGB could suppress rat mesangial cells hypertrophy and ECM accumulation through decreasing Smad2/3 and TGF- $\beta 1$ and increasing Smad7 [73], while in DN patients EGB has been proven to retard early DN development through decreasing serum sICAM-1 and sVCAM-1 levels [72].

2.2.7. Flos Abelmoschus manihot. Flos Abelmoschus manihot (Huangshukuihua in Chinese) has been widely used as the neuroprotective drug for cerebral ischemic reperfusion injury. Total flavone glycosides of flos A. manihot (TFA) contain 7 identified flavone glycosides. TFA pretreatment has been shown to prevent renal damage and podocyte apoptosis in STZ induced rats [76]. A meta-analysis of 27 randomized controlled trials showed that flos Abelmoschus manihot had significant effect on renal function in the treatment of $\mathrm{DN}$ deserving further investigation [115].

2.2.8. Genipin. Genipin is a glycone derived from geniposide present in fruit of Gardenia jasminoides. Genipin has been proven to ameliorate body weight loss and urine albumin leakage, attenuate GBM thickness, and restore the podocyte expression of podocin and WT1 in diabetic mice; the protective effect of Genipin on DN is probably through suppressing the upregulation of mitochondrial UCP2 in STZ induced diabetic mice kidneys [77].

\subsubsection{Houttuynia cordata Thunb. (HCT). Houttuynia cordata} Thunb. (HCT, Yu Xing Cao in Chinese), pungent in taste and cool in nature, has been reported to reduce urinary proteins in the patients with nephrotic syndrome; HCT has also been shown to protect diabetic kidney function through decreasing the expression of TGF- $\beta 1$ and increasing the expression of BMP-7 [78].

2.2.10. Icariin. Icariin is a major constituent of flavonoid extracted from the plant herba epimedii and has been shown to relieve renal damage in STZ induced diabetic rats through inhibiting the expression of TGF- $\beta 1$ and collagen IV protein [79].

2.2.11. $L A B$. Lithospermate $B(L A B)$, a tetramer of caffeic acid isolated from Salvia miltiorrhiza radix, was identified as antioxidant and PKC inhibitor in the renoprotective effects under diabetic conditions in vivo and in vitro [80]. In the STZ induced diabetic rats, delayed LAB treatment could inhibit renal MDA, microalbuminuria, mesangial expansion, and glomerular hypertrophy, and in mesangial cells LAB could inhibit $\mathrm{HG}$ and $\mathrm{H}_{2} \mathrm{O}_{2}$ induced TGF- $\beta 1$ and FN secretion, HG induced intracellular PKC activation, and ROS generation, which suggested that $\mathrm{LAB}$ could significantly suppress the progression of diabetic renal injury. A recent study showed that LAB could prevent diabetic atherosclerosis by induction of the Nrf2-ARE-NQO1 pathway to inhibit VSMCs proliferation and migration and vascular damage [81]. All these findings suggested that $\mathrm{LAB}$ could be a new therapeutic agent in the treatment of DN. In the subsequent study, Salvia miltiorrhiza could protect STZ induced diabetic rats by inhibiting the overexpression of TGF- $\beta 1$, CTGF, PAI-1, and FN in renal cortex.

2.2.12. LBP. Lycium barbarum polysaccharide (LBP) is extracted from the fruit of goji berry(Solanaceae); LBP4 
has been shown to protect STZ induced diabetic kidney function via decreasing the activation of ERK1/2 through the involvement of PKC in mesangial cells [82].

2.2.13. LGP. Lyoniresinol 3 alpha-O-beta-D-glucopyranoside (LGP) is isolated from Averrhoa carambola L. (Oxalidaceae) root (ACLR), including two chiral lignin glucosides: LGP1 and LGP2. LGP1 treatment has been shown to decrease hyperglycemia and the expression of related proteins including NF- $\kappa \mathrm{B}$, caspase-3, caspase-8, caspase-9, and Bax in STZ induced diabetic mice. LGP1 also could alleviate glomerular hypertrophy, excessive ECM accumulation, and glomerular and tubular basement membrane thickness. All these data suggested that LGP1 could be a potential therapeutic agent in $\mathrm{DN}[83]$.

2.2.14. Ligustrazine. Ligustrazine, a bioactive component of Chuangxiong, has been widely used in the treatment of vascular diseases such as myocardial and cerebral infarction in China. A meta-analysis of 25 studies showed that Ligustrazine has therapeutic effect to improve renal function and reduce urine protein excretion in DN patients [84]. Further studies should be conducted to reveal the underlying mechanism for the treatment on DN.

2.2.15. MC. Moutan cortex (MC), the root bark of Paeonia suffruticosa, has been shown to have the protective effect against atherosclerosis and inflammation and inhibitory effect on the production of ROS. MC was reported to increase activity of SOD, GSH-PX, and CAT and reduce MDA in vitro or in vivo; furthermore, MC could decrease blood glucose, Scr, and urine protein in HFD-fed plus STZ induced diabetic rats, which suggested that $\mathrm{MC}$ has renal protective effect in AGEs-induced mesangial cell dysfunction through attenuating oxidative stress pathway [85], while, in AGEs-induced rat mesangial cells, MC could inhibit FN and collagen IV expression in matrix [86]. Apart from the abovementioned evidence of renal protective effect on $\mathrm{DN}$, MC could ameliorate activity on the inflammation via target of RAGE in vitro or in vivo [87].

2.2.16. Morroniside. Corni fructus, a constituent of HJG, used as a traditional medicine in China and Japan, has been shown to be superior to aminoguanidine treatment in suppressing hyperglycemia, proteinuria, renal AGE formation, and TGF$\beta 1$ expression in STZ induced diabetic rats [116]. Morroniside, isolated from corni fructus, could exhibit protective effects against STZ induced renal damage by inhibiting hyperglycemia and oxidative stress [88]. Another study showed that components of corni fructus could play protective effect on early stage of DN in type 2 diabetic rats mediated by the regulation of podocytes. Loganin from corni fructus and its derivatives could inhibit the expression of FN and IL-6 in the HG stimulated mesangial cells, which supported the traditional use of corni fructus in DN and relevant kidney diseases [117].

2.2.17. Panax Notoginoside (PNS). Panax notoginoside (PNS) is extracted from radix notoginseng and has been shown to protect kidney in type 1 diabetic rats at early stage through inhibiting the expression of VEGF protein and enhancing BMP-7 expression in the kidney [89]. Another report showed that the protective effect of PNS in kidney was mediated by inhibiting TGF- $\beta 1$ expression and enhancing the expression of Smad7 [90]. Ginsenoside Rg1, an active ingredient isolated from PNS, has been shown to improve the renal pathological changes in STZ induced diabetic rats through reducing TGF$\beta 1$ expression and inflammatory reaction factors including CRP and TNF- $\alpha$ [118]. Ginsenoside Rg1 also could effectively relieve aldosterone-induced oxidative stress through which it indirectly inhibits aldosterone-induced podocyte autophagy [119].

2.2.18. Puerarin. Puerarin, 7-hydroxy-3-(4-hydroxyphenyl)1-benzopyran-4-one-8-b-D-glucopyranosid-e, is one of the major isoflavonoid compounds from the root of Pueraria candollei wall of Leguminosae family. A previous study showed that Puerarin could protect DN rats by inhibiting collagen IV expression [91]; further study in STZ induced diabetic rats showed that Puerarin could protect kidney function through downregulating MMP-9 and attenuating eNOS expression $[92,93]$.

2.2.19. Rehmannia Radix (Di Huang). Rehmannia radix (Di Huang) was mostly mentioned and investigated; it has been proven to reduce hyperglycemia, ameliorate renal dysfunction, prevent senility, and improve hemorheology. In a previous experimental study Rehmannia radix has been shown to inhibit the progression of DN [120]. Catalpol is an iridoid glucoside compound mainly present in Rehmannia radix and other plants amd has been shown to reduce ECM accumulation by inhibiting the expression of TGF- $\beta 1$, CTGF, and Ang II in HFD-fed plus STZ induced diabetic rats [121].

2.2.20. Rhein. Rhein (4,5-dihydroxyanthraquinone-2-carboxylic acid) is purified from rhubarb (Rheum officinale). Rhein has shown reduction of UAE faster than simvastatin and decrease of ECM levels along with decreased TGF- $\beta 1$ and FN immunohistochemistry expression in $\mathrm{db} / \mathrm{db}$ renal tissue, which was supposed via regulation of dyslipidemia [94]. Another study showed that Rhein could inhibit the hypertrophy of rat renal proximal tubular epithelial cells stimulated by HG and Ang II [95].

2.2.21. Rhodiola rosea. Rhodiola rosea ( $R$. rosea) is grown at northern latitudes and high altitudes of the world; Rhodiola rosea extract has been used to protect kidney function including reducing $\mathrm{FBG}, \mathrm{TC}, \mathrm{TG}, \mathrm{Ccr}$, and $24 \mathrm{~h}$ urinary albumin in HFD-fed plus STZ induced diabetic rats through decreasing renal expression of TGF- $\beta 1$ [96].

2.2.22. Rosa laevigata Michx. (RLM). Rosa laevigata Michx. (RLM), a commonly used TCM for the treatment of urinary tract infection and antioxidative treatment, could play a critical role in the pathogenesis of DN through increasing the activity of SOD and total antioxidant capacity, decreasing MDA and ROS levels, and inhibiting NF- $\kappa$ B p 65 and MCP-1 expression following increased $\mathrm{I} \kappa \mathrm{B}$ protein expression in $\mathrm{STZ}$ 
induced diabetic rats; all the data suggested that RLM could be a therapeutic potential for DN [97].

2.2.23. Sequoyitol. Sequoyitol is a natural compound present in a lot of plants (e.g., Aristolochia arcuata, Amentotaxus yunnanensis, and Crossostephium chinensis); oral and subcutaneous administrations of sequoyitol could ameliorate hyperglycemia and glucose intolerance in ob/ob mice. Sequoyitol has been shown to ameliorate the progression of DN in HFDfed plus STZ induced rats through glucose-lowering effects, antioxidant activity, and regulation of TGF- $\beta 1$ expression [98].

2.2.24. SF/FA. Sodium ferulate (SF), extracted from Angelica sinensis, Lignsticum chuangxiong, Cimicifuga heracleifolia, and other plants, has platelet aggregation inhibitory, antithrombotic, and antioxidant activities in animals and humans. A preliminary study on DN patients showed that SF could lower UAER level and improve renal function through decreasing endothelin (ET) and inhibiting the combination of ET with its receptor [99]. A meta-analysis of 14 randomized controlled trials involving 906 patients showed that SF is superior in reducing UAER, ET, BUN, Scr, and TC and increasing HDL-c without affecting FBG and TG [122]. Ferulic acid (FA) is a phenolic acid extracted from the seeds and leaves of most plants and has antioxidant activities, hypoglycemic and hypolipidemic effects, hypotensive effects, and anti-inflammatory effects. In the FA treated OLETF rats, blood glucose and urinary ACR were decreased significantly; in renal histopathology glomerular basement membrane thickness and mesangial matrix expansion were decreased through reducing oxidative stress and inflammation $[74,75]$.

2.2.25. Skimmin. Skimmin, a major active component from Hydrangea paniculata, has been reported to decrease Scr and blood glucose level and alleviate glomerular segmental sclerosis and incidence of tubular vacuolar degeneration by downregulating the TGF- $\beta 1$ and TGF- $\beta 1$ receptor I expression in STZ induced diabetic rats [100].

2.2.26. SM. Salvia miltiorrhiza (SM, commonly known as Danshen in Chinese) has been shown to have the antiinflammatory, antioxidative, and organ protective effects. A previous study showed that SM could protect STZ induced diabetic rats from $\mathrm{DN}$ by suppressing the overexpression of TGF- $\beta 1$, CTGF, PAI-1, and FN in renal cortex [101]. Another study showed that SM could ameliorate TGF- $\beta 1$ levels in serum and kidney and reduce the levels of collagen IV ED1 and RAGE in the diabetic kidney [102]. Danshen injection, the aqueous extracts of SM, could protect diabetic rats associated with preservation of tubular function and structure from hyperglycemia induced oxidative stress, advanced glycation stress, and megalin expression deletion [103].

2.2.27. TGP. Total glucosides of paeony (TGP), extracted from the root of Paeonia lactiflora Pall., have been shown to have the therapeutic effect in the experimental DN. TGP treatment in the STZ induced diabetic rats could prevent diabetic renal damage against oxidative stress through decreasing upregulated p-p38 MAPK and NF- $\kappa$ B P65 expressions [104]. And, in the HFD-fed plus STZ induced rats, TGP could improve kidney damage and delay the development of $\mathrm{DN}$ by inhibiting Wnt/beta-catenin signaling pathway [105].

2.2.28. TMP. Tetramethylpyrazine (TMP) is isolated from Ligusticum chuanxiong and has been used in the treatment of stroke and cardiovascular diseases. TMP was reported to reduce diabetic kidney damage partially by downregulating the expression of VEGF in the kidney [106].

2.2.29. Triptolide/GTW/TwHF. Triptolide, active diterpene purified from Tripterygium wilfordii Hook. F. (TwHF), has been reported to have anti-inflammatory, antioxidative, immunosuppressive, and podocyte-protective effects. A recent study showed that triptolide could attenuate albuminuria in $\mathrm{db} / \mathrm{db}$ diabetic mice accompanied with alleviated glomerular hypertrophy and podocyte injury, while inflammation and dyslipidemia were also attenuated [107]. Triptolide is one of the major active components of multiglycoside of TwHF (GTW), and GTW has been applied extensively for the treatment of CKD in China as an anti-inflammatory agent. GTW could prevent glomerular lesion in STZ induced diabetic model through decreasing urine albumin and ameliorating glomerular sclerosis [123]. A recent study showed that TwHF could prevent podocyte injury of DN patients, which may be partly mediated by downregulating the expression of OPN, CTGF, and TGF- $\beta 1$ [108].

2.2.30. Volatile Oil of Magnolia biondii Pamp. (VOMBP). Volatile oil of Magnolia biondii Pamp. (VOMBP), extracted from herbal TCM Magnolia biondii Pamp., has been reported to protect the kidney in STZ induced diabetic rats by inhibiting the expression of P-selectin in serum and renal tissue [109].

2.3. TCMs Combined Therapy with Western Medicines in $D N$. Apart from the TCM preparations and single TCM applications in DN, TCMs combined with western medicines have been indicated. Mostly used western medicines were ACEI/ARBs, and combination styles included Tangshenling (TSL) with telmisartan [124] in diabetic patients or TSL with benazepril in STZ induced rats [125], triptolide with benazepril in DN patients [126], Bailing Capsule (BC) and benazepril in DN patients [127], and safflower yellow powder injection with benazepril in DN patients [128]. Another report is about Tangshenqing (TSQ) combined with alprostadil in the treatment of DN patients [129]. All data suggested that effects of TCMs combined therapy with western medicines were superior to western medicines treatment alone.

\section{Conclusions and Perspectives}

Although there are almost no side effects mentioned in numerous scientific reports, a lot of scientific researches indicate that herbal TCM preparations have renal protective 
effects on DN according to respective factors, complexity, and variability of TCM preparations still presenting challenges for clinicians seeking scientific evidence to support TCM application in drug discovery. In order to avoid the toxicity and side effects of TCM formulas, there is increasing interest in studying single herbal TCM especially monomers from single herbal TCM on DN. In this review, we found that monomers such as Berberine, curcumin, Ginsenoside Rg1, Puerain, Rhein, and Ferulic acid have specific protective effect on DN. To translate the therapeutic potentials for DN into reality, placebo-controlled and randomized controlled clinical trials of single herbal TCM and/or monomers from herbal TCM are essential in the future, and prompt metaanalysis is an effective alternative.

\section{Conflict of Interests}

The authors have no conflict of interests to declare.

\section{References}

[1] T. Scully, "Diabetes in numbers," Nature, vol. 485, no. 7398, pp. 52-53, 2012.

[2] E. Vivian and C. Mannebach, "Therapeutic approaches to slowing the progression of diabetic nephropathy-is less best?" Drugs in Context, vol. 2013, Article ID 212249, 2013.

[3] R. J. Macisaac, E. I. Ekinci, and G. Jerums, "Markers of and risk factors for the development and progression of diabetic kidney disease," American Journal of Kidney Diseases, vol. 63, no. 2, pp. S39-S62, 2014.

[4] J. Skupien, J. H. Warram, A. M. Smiles et al., "The early decline in renal function in patients with type 1 diabetes and proteinuria predicts the risk of end-stage renal disease," Kidney International, vol. 82, no. 5, pp. 589-597, 2012.

[5] Y. S. Kanwar, L. Sun, P. Xie, F.-Y. Liu, and S. Chen, "A glimpse of various pathogenetic mechanisms of diabetic nephropathy," Annual Review of Pathology: Mechanisms of Disease, vol. 6, pp. 395-423, 2011.

[6] M. A. Reddy, J. Tak Park, and R. Natarajan, "Epigenetic modifications in the pathogenesis of diabetic nephropathy," Seminars in Nephrology, vol. 33, no. 4, pp. 341-353, 2013.

[7] S. O. Kolset, F. P. Reinholt, and T. Jenssen, "Diabetic nephropathy and extracellular matrix," The Journal of Histochemistry and Cytochemistry, vol. 60, no. 12, pp. 976-986, 2012.

[8] G. D. Sun, W. P. Cui, Q. Y. Guo, and L. N. Miao, "Histone lysine methylation in diabetic nephropathy," Journal of Diabetes Research, vol. 2014, Article ID 654148, 9 pages, 2014.

[9] A. P. Sanchez and K. Sharma, "Transcription factors in the pathogenesis of diabetic nephropathy," Expert Reviews in Molecular Medicine, vol. 11, article e13, 2009.

[10] A. K. H. Lim, "Diabetic nephropathy-complications and treatment," International Journal of Nephrology and Renovascular Disease, vol. 7, pp. 361-381, 2014.

[11] H. J. L. Heerspink and D. de Zeeuw, "The kidney in type 2 diabetes therapy," Review of Diabetic Studies, vol. 8, no. 3, pp. 392-402, 2011.

[12] H. Yamout, I. Lazich, and G. L. Bakris, "Blood pressure, hypertension, RAAS blockade, and drug therapy in diabetic kidney disease," Advances in Chronic Kidney Disease, vol. 21, no. 3, pp. 281-286, 2014.
[13] L. F. Fried, N. Emanuele, J. H. Zhang et al., "Combined angiotensin inhibition for the treatment of diabetic nephropathy," The New England Journal of Medicine, vol. 369, no. 20, pp. 1892-1903, 2013.

[14] X.-L. Tong, L. Dong, L. Chen, and Z. Zhen, "Treatment of diabetes using traditional Chinese medicine: past, present and future," The American Journal of Chinese Medicine, vol. 40, no. 5, pp. 877-886, 2012.

[15] X. Shi, X. G. Lu, L. B. Zhan et al., "The effects of the Chinese medicine $\mathrm{ZiBu}$ PiYin recipe on the hippocampus in a rat model of diabetes-associated cognitive decline: a proteomic analysis," Diabetologia, vol. 54, no. 7, pp. 1888-1899, 2011.

[16] H.-L. Zhao, Y. Sui, C.-F. Qiao et al., "Sustained antidiabetic effects of a berberine-containing Chinese herbal medicine through regulation of hepatic gene expression," Diabetes, vol. 61, no. 4, pp. 933-943, 2012.

[17] X. Wen, Y. Zeng, L. Liu et al., "Zhenqing recipe alleviates diabetic nephropathy in experimental type 2 diabetic rats through suppression of SREBP-1c," Journal of Ethnopharmacology, vol. 142, no. 1, pp. 144-150, 2012.

[18] R. Teschke, A. Wolff, C. Frenzel, A. Eickhoff, and J. Schulze, "Herbal traditional Chinese medicine and its evidence base in gastrointestinal disorders," World Journal of Gastroenterology, vol. 21, no. 15, pp. 4466-4490, 2015.

[19] G. Wang, B. Mao, Z.-Y. Xiong et al., "The quality of reporting of randomized controlled trials of traditional Chinese medicine: a survey of 13 randomly selected journals from mainland China," Clinical Therapeutics, vol. 29, no. 7, pp. 1456-1467, 2007.

[20] T. T. Zhao, H. J. Zhang, X. G. Lu et al., "Chaihuang-Yishen granule inhibits diabetic kidney disease in rats through blocking TGF- $\beta /$ Smad3 signaling," PLoS ONE, vol. 9, no. 3, Article ID e90807, 2014.

[21] S. Liu, L.-Q. Tang, L.-M. Chen, X.-Q. Chen, S.-T. Zhang, and Y. Wei, "Effect of compound Rhizoma Coptidis capsule on expression of transforming growth factor- $\beta 1$ and type IV collagen proteins in renal tissue of diabetic rats with nephropathy," Zhongguo zhongyao zazhi, vol. 33, no. 1, pp. 68-72, 2008.

[22] W.-J. Geng, R.-B. Wei, and W. Mao, "Protective effects of compound shenhua tablet on diabetic nephropathy rats," Zhongguo Zhongxiyi Jiehe Zazhi, vol. 32, no. 3, pp. 352-355, 2012.

[23] Y. W. Zhang, D. Xie, B. Xia, R. T. Zhen, I.-M. Liu, and J.T. Cheng, "Suppression of transforming growth factor- $\beta_{1}$ gene expression by danggui buxue tang, a traditional Chinese herbal preparation, in retarding the progress of renal damage in streptozotocin-induced diabetic rats," Hormone and Metabolic Research, vol. 38, no. 2, pp. 82-88, 2006.

[24] H.-L. Ke, Y.-W. Zhang, B.-F. Zhou, and R.-T. Zhen, "Effects of Danggui Buxue Tang, a traditional Chinese herbal decoction, on high glucose-induced proliferation and expression of extracellular matrix proteins in glomerular mesangial cells," Natural Product Research, vol. 26, no. 11, pp. 1022-1026, 2012.

[25] I.-M. Liu, T.-F. Tzeng, S.-S. Liou, and C. J. Chang, "Beneficial effect of traditional chinese medicinal formula DangguiShaoyao-San on advanced glycation end-product-mediated renal injury in streptozotocin-diabetic rats," Evidence-Based Complementary and Alternative Medicine, vol. 2012, Article ID 140103, 10 pages, 2012.

[26] D. Fang, X. Wan, W. Deng et al., "Fufang Xue Shuan tong capsules inhibit renal oxidative stress markers and indices of nephropathy in diabetic rats," Experimental and Therapeutic Medicine, vol. 4, no. 5, pp. 871-876, 2012. 
[27] T. Yokozawa, N. Yamabe, E. J. Cho, T. Nakagawa, and S. Oowada, "A study on the effects to diabetic nephropathy of hachimi-jiogan in rats," Nephron Experimental Nephrology, vol. 97, no. 2, pp. e38-e48, 2004.

[28] C. Kiga, T. Nakagawa, K. Koizumi et al., "Expression patterns of plasma proteins in spontaneously diabetic rats after oral administration of a Kampo medicine, Hachimi-jio-gan, using SELDI ProteinChip platform," Biological \& Pharmaceutical Bulletin, vol. 28, no. 6, pp. 1031-1037, 2005.

[29] T. Nakagawa, T. Yokozawa, N. Yamabe et al., "Long-term treatment with Hachimi-jio-gan attenuates kidney damage in spontaneously diabetic WBN/Kob rats," Journal of Pharmacy and Pharmacology, vol. 57, no. 9, pp. 1205-1212, 2005.

[30] N. Yamabe and T. Yokozawa, "Activity of the Chinese prescription Hachimi-jio-gan against renal damage in the Otsuka LongEvans Tokushima fatty rat: a model of human type 2 diabetes mellitus,", The Journal of Pharmacy and Pharmacology, vol. 58, no. 4, pp. 535-545, 2006.

[31] L. Zhou, H. Dong, Y. Huang et al., "Hu-Lu-Ba-Wan attenuates diabetic nephropathy in type 2 diabetic rats through PKC$\alpha / \mathrm{NADPH}$ oxidase signaling pathway," Evidence-Based Complementary and Alternative Medicine, vol. 2013, Article ID 504642, 10 pages, 2013.

[32] X.-Y. Song, Q. Chen, and X.-Y. Qi, "Effect of liuwei dihuang pill on erythrocyte aldose reductase activity in early diabetic nephropathy patients," Zhongguo Zhongxiyi Jiehe Zazhi, vol. 24, no. 12, pp. 1087-1090, 2004.

[33] P.-C. Hsu, Y.-T. Tsai, J.-N. Lai, C.-T. Wu, S.-K. Lin, and C.-Y. Huang, "Integrating traditional Chinese medicine healthcare into diabetes care by reducing the risk of developing kidney failure among type 2 diabetic patients: a population-based case control study," Journal of Ethnopharmacology, vol. 156, pp. 358364, 2014.

[34] I.-M. Liu, T.-F. Tzeng, S.-S. Liou, and C. J. Chang, “The amelioration of streptozotocin diabetes-induced renal damage by Wu-Ling-San (Hoelen Five Herb Formula), a traditional Chinese prescription," Journal of Ethnopharmacology, vol. 124, no. 2, pp. 211-218, 2009.

[35] J. J. Yoon, Y. J. Lee, D. G. Kang, and H. S. Lee, "Protective role of oryeongsan against renal inflammation and glomerulosclerosis in $\mathrm{db} / \mathrm{db}$ mice," The American Journal of Chinese Medicine, vol. 42, no. 6, pp. 1431-1452, 2014.

[36] Z. A. Guo, C. J. Yu, G. Liu, F. C. Meng, Y. Li, and S. L. Peng, "Treatment of stage $3 \mathrm{~b}$ diabetic kidney disease patients with macroalbuminuria by Qizhi Jiangtang capsule: a multicenter randomized control clinical study," Zhongguo Zhongxiyi Jiehe Zazhi, vol. 34, no. 9, pp. 1047-1052, 2014.

[37] M.-Z. Li, Y.-B. Gao, and M.-F. Ma, "Effects of qiwei granule on the protein and mRNA expressions of renal tissue transforming growth factor-betal in KK-Ay mice with spontaneous type 2 diabetes mellitus," Zhongguo Zhongxiyi jiehe zazhi, vol. 32, no. 12, pp. 1675-1678, 2012.

[38] G.-B. Chen, L.-B. Wei, W. Xiao, and H.-B. Long, "Effect of Shenkangwan on mesangial cell NO and TGF-betal excretion in rats with early diabetic nephropathy," Journal of Southern Medical University, vol. 26, no. 4, pp. 526-528, 2006.

[39] H.-B. Long, H.-X. Niu, X.-Y. Li et al., "Effects of Shenkangwan on renal expressions of angiotensin II and its type I receptor in rats with early diabetic nephropathy," Journal of Southern Medical University, vol. 30, no. 4, pp. 805-809, 2010.

[40] J.-J. Yin, Y. Yang, Q.-B. Wang, Y. Li, and D.-K. Yin, "Effect of Chinese herbal medicine with Supplement Qi and Activating
Blood Circulation on tubular reabsorption function of diabetic nephropathy rats," Journal of Chinese medicinal materials, vol. 36, no. 6, pp. 953-958, 2013.

[41] D.-K. Yin, Y. Yang, and Y. Li, "Effect of supplementing Qi and activating blood circulation method on protein uptake in NRK52 E cells injured by high glucose," Journal of Chinese Medicinal Materials, vol. 37, no. 1, pp. 91-94, 2014.

[42] M. Huang, C. Zhu, Q.-L. Liang et al., "Effect of Tangshen formula on phospholipids metabolism in diabetic nephropathy patients," Acta Pharmaceutica Sinica, vol. 46, no. 7, pp. 780-786, 2011.

[43] Z.-T. Jiang, Q.-L. Liang, and Y.-M. Wang, "Effects of tangshen recipe on the homocysteine metabolism of patients with diabetic nephropathy," Zhongguo Zhongxiyi jiehe zazhi, vol. 31, no. 8, pp. 1057-1061, 2011.

[44] J. Hu, X. Fan, X. Meng, Y. Wang, Q. Liang, and G. Luo, "Evidence for the involvement of JAK/STAT/SOCS pathway in the mechanism of Tangshen formula-treated diabetic nephropathy," Planta Medica, vol. 80, no. 8-9, pp. 614-621, 2014.

[45] Y.-L. Wu, C. Wei, and H.-T. Wang, "Effect of tongshenluo capsule on the components of extracellular matrix and their metabolism in kidney of rats with diabetic nephropathy," Zhongguo Zhongxiyi Jiehe Zazhi, vol. 27, no. 4, pp. 326-330, 2007.

[46] Y. Zhao and X.-L. Zhang, "Effect of tongxinluo capsule on plasma endothelin in patients with diabetic nephropathy," Zhongguo Zhongxiyi Jiehe Zazhi, vol. 25, no. 2, pp. 131-133, 2005.

[47] J.-Y. Wang, Y.-B. Gao, N. Zhang et al., "Tongxinluo ameliorates renal structure and function by regulating mir-21-induced epithelial-to-mesenchymal transition in diabetic nephropathy," The American Journal of Physiology — Renal Physiology, vol. 306, no. 5, pp. F486-F495, 2014.

[48] C.-C. Lin, L.-T. Lin, M.-H. Yen, J.-T. Cheng, C.-H. Hsing, and C.-H. Yeh, "Renal protective effect of Xiao-Chai-Hu-Tang on diabetic nephropathy of type 1-diabetic mice," Evidence-Based Complementary and Alternative Medicine, vol. 2012, Article ID 984024, 11 pages, 2012.

[49] X.-M. Ji, Q. Wang, and F. Qi, "Effect of xiaoke granule on blood glucose, urinary protein and glomerular morphology in rats with diabetic nephropathy," Zhongguo Zhongxiyi Jiehe Zazhi, vol. 24, no. 6, pp. 534-537, 2004.

[50] X.-M. Ji, Q. Wang, M.-X. Gong, Y.-Q. Du, and D.-X. Jia, "Effects of Xiaoke granule on transforming growth factor-betal, expression and proliferation in rat mesangial cells," Chinese Medical Journal, vol. 119, no. 21, pp. 1839-1842, 2006.

[51] J.-S. Wu, R. Shi, J. Zhong et al., "Renal protective role of Xiexin decoction with multiple active ingredients involves inhibition of inflammation through downregulation of the nuclear factor- $\kappa \mathrm{B}$ pathway in diabetic rats," Evidence-Based Complementary and Alternative Medicine, vol. 2013, Article ID 715671, 15 pages, 2013.

[52] J. S. Wu, R. Shi, X. Lu, Y. M. Ma, and N. N. Cheng, "Combination of active components of xiexin decoction ameliorates renal fibrosis through the inhibition of NF- $\kappa \mathrm{B}$ and TGF- $\beta 1 / \mathrm{smad}$ pathways in $\mathrm{db} / \mathrm{db}$ diabetic mice," PLoS ONE, vol. 10, no. 3, Article ID e0122661, 2015.

[53] D.-Y. Tang, S.-S. Guo, and R.-Y. Sun, "Effect of xianzhen tablet on content of advanced glycosylation end products (AGEs) and mRNA expression of AGE-specific cellular receptor in renal cortex of diabetic rats," Zhongguo Zhongxiyi jiehe zazhi, vol. 25, no. 1, pp. 60-63, 2005.

[54] L. Zhao, H. Gao, Y. Zhao, and D. Lin, "Metabonomic analysis of the therapeutic effect of Zhibai Dihuang Pill in treatment 
of streptozotocin-induced diabetic nephropathy," Journal of Ethnopharmacology, vol. 142, no. 3, pp. 647-656, 2012.

[55] J. W. Ju, S. H. Mu, Y. Z. Yin, C. K. Lu, X. Y. Sun, and L. X. Deng, "Effects of zao huang mixture (see text) on the expressions of TGF-betal and Col IV in human glomerular mesangial cells cultured in high glucose environment," Journal of Traditional Chinese Medicine, vol. 31, no. 2, pp. 127-129, 2011.

[56] J. Ma, L. Xu, J. Dong et al., "Effects of zishentongluo in patients with early-stage diabetic nephropathy," The American Journal of Chinese Medicine, vol. 41, no. 2, pp. 333-340, 2013.

[57] H. He, X. Yang, X. Zeng et al., "Protective effect of Liuwei Dihuang decoction on early diabetic nephropathy induced by streptozotocin via modulating ET-ROS axis and matrix metalloproteinase activity in rats," The Journal of Pharmacy and Pharmacology, vol. 59, no. 9, pp. 1297-1305, 2007.

[58] H.-R. Liu, X.-Y. Tang, D.-Z. Dai, and Y. Dai, "Ethanol extracts of Rehmannia complex (Di Huang) containing no Corni fructus improve early diabetic nephropathy by combining suppression on the ET-ROS axis with modulate hypoglycemic effect in rats," Journal of Ethnopharmacology, vol. 118, no. 3, pp. 466-472, 2008.

[59] W. Xiao, L.-B. Wei, Y. Ma, H.-B. Long, and G.-B. Chen, "Renal protective effect of Shenkang pill on diabetic rats," Zhongguo zhongyao zazhi, vol. 31, no. 12, pp. 1006-1009, 2006.

[60] W. Xiao, Y. Ma, and L.-B. Wei, "Effect of shenkangwan on TGFbetal and FN in rat mesangial cells cultured by high glucose," Journal of Chinese Medicinal Materials, vol. 29, no. 9, pp. 924927, 2006.

[61] H.-B. Long, H. Zhang, J. Zhong, Y. Zhu, J.-H. He, and L.-B. Wei, "Protective effects of Shenkangwan against podocyte injury in rats with early diabetic nephropathy," Journal of Southern Medical University, vol. 28, no. 7, pp. 1268-1272, 2008.

[62] N. Zhang, Y. Gao, D. Zou et al., "Effects of Chinese medicine Tong xinluo on diabetic nephropathy via inhibiting TGF- $\beta 1-$ induced epithelial-to-mesenchymal transition," Evidence-Based Complementary and Alternative Medicine, vol. 2014, Article ID 123497, 12 pages, 2014.

[63] M. Li, W. Wang, J. Xue, Y. Gu, and S. Lin, "Meta-analysis of the clinical value of Astragalus membranaceus in diabetic nephropathy," Journal of Ethnopharmacology, vol. 133, no. 2, pp. 412-419, 2011.

[64] Y. Nie, S. Li, Y. Yi et al., "Effects of astragalus injection on the TGF $\beta /$ Smad pathway in the kidney in type 2 diabetic mice," BMC Complementary and Alternative Medicine, vol. 14, article 148, 2014.

[65] S. Mou, Z.-H. Ni, and Q.-Y. Zhang, "Expression of c-met in human kidney fibroblasts induced by high glucose in vitro and the regulation of Radix Astragali," Journal of Chinese Integrative Medicine, vol. 6, no. 5, pp. 482-487, 2008.

[66] F. L. Wang, L. Q. Tang, F. Yang, L. N. Zhu, M. Cai, and W. Wei, "Renoprotective effects of berberine and its possible molecular mechanisms in combination of high-fat diet and lowdose streptozotocin-induced diabetic rats," Molecular Biology Reports, vol. 40, no. 3, pp. 2405-2418, 2013.

[67] M. Lu, L. Tao, W. Mei et al., "Effect of curcumin on the expression of p-STAT3 and $\mathrm{I} \kappa \mathrm{B}$ in $\mathrm{db} / \mathrm{db}$ mice," Journal of Central South University (Medical Sciences), vol. 39, no. 6, pp. 591-597, 2014.

[68] J.-P. Liu, L. Feng, M.-M. Zhu et al., “The in vitro protective effects of curcumin and demethoxycurcumin in Curcuma longa extract on advanced glycation end products-induced mesangial cell apoptosis and oxidative stress," Planta Medica, vol. 78, no. 16, pp. 1757-1760, 2012.
[69] N. Zheng, X. Lin, Q. Wen et al., "Effect of 2-dodecyl-6-methoxycyclohexa-2,5-diene-1,4-dione, isolated from Averrhoa carambola L. (Oxalidaceae) roots, on advanced glycation endproduct-mediated renal injury in type 2 diabetic KKAy mice," Toxicology Letters, vol. 219, no. 1, pp. 77-84, 2013.

[70] Y. Xie, Q. Wang, J. Liu, J. Xie, K. Xue, and Q. Tang, "Dracorhodin perchlorate inhibit high glucose induce serum and glucocorticoid induced protein kinase 1 and fibronectin expression in human mesangial cells," Zhongguo Zhongyao Zazhi, vol. 35, no. 15, pp. 1996-2000, 2010.

[71] H.-W. Zhu, Z.-F. Shi, and Y.-Y. Chen, "Effect of extract of ginkgo bilboa leaf on early diabetic nephropathy," Zhongguo Zhongxiyi jiehe zazhi, vol. 25, no. 10, pp. 889-891, 2005.

[72] X.-S. Li, X.-J. Fu, and X.-J. Lang, "Effect of extract of Gingko biloba on soluble intercellular adhesion molecule-1 and soluble vascular cell adhesion molecule-1 in patients with early diabetic nephropathy," Zhongguo Zhongxiyi Jiehe Zazhi, vol. 27, no. 5, pp. 412-414, 2007.

[73] J.-Y. Wang, X.-X. Yin, Y.-M. Wu et al., "Ginkgo biloba extract suppresses hypertrophy and extracellular matrix accumulation in rat mesangial cells," Acta Pharmacologica Sinica, vol. 27, no. 9, pp. 1222-1230, 2006.

[74] A. Fujita, H. Sasaki, A. Doi et al., "Ferulic acid prevents pathological and functional abnormalities of the kidney in Otsuka Long-Evans Tokushima Fatty diabetic rats," Diabetes Research and Clinical Practice, vol. 79, no. 1, pp. 11-17, 2008.

[75] R. Choi, B. H. Kim, J. Naowaboot et al., "Effects of ferulic acid on diabetic nephropathy in a rat model of type 2 diabetes," Experimental \& Molecular Medicine, vol. 43, no. 12, pp. 676-683, 2011.

[76] L. Zhou, X.-F. An, S.-C. Teng et al., "Pretreatment with the total flavone glycosides of Flos Abelmoschus manihot and hyperoside prevents glomerular podocyte apoptosis in streptozotocininduced diabetic nephropathy," Journal of Medicinal Food, vol. 15, no. 5, pp. 461-468, 2012.

[77] W. Qiu, Y. Zhou, L. Jiang et al., "Genipin inhibits mitochondrial uncoupling protein 2 expression and ameliorates podocyte injury in diabetic mice," PLoS ONE, vol. 7, no. 7, Article ID e41391, 2012.

[78] F. Wang, F. Lu, and L. Xu, "Effects of Houttuynia cordata Thumb on expression of BMP-7 and TGF- $\beta_{1}$ in the renal tissues of diabetic rats," Journal of Traditional Chinese Medicine, vol. 27, no. 3, pp. 220-225, 2007.

[79] M.-Y. Qi, Kai-Chen, H.-R. Liu, Y.-H. Su, and S.-Q. Yu, "Protective effect of Icariin on the early stage of experimental diabetic nephropathy induced by streptozotocin via modulating transforming growth factor $\beta_{1}$ and type IV collagen expression in rats," Journal of Ethnopharmacology, vol. 138, no. 3, pp. 731736, 2011.

[80] G. T. Lee, H. Ha, M. Jung et al., "Delayed treatment with lithospermate B attenuates experimental diabetic renal injury," Journal of the American Society of Nephrology, vol. 14, no. 3, pp. 709-720, 2003.

[81] K. Y. Hur, S. H. Kim, M.-A. Choi et al., "Protective effects of magnesium lithospermate $\mathrm{B}$ against diabetic atherosclerosis via Nrf2-ARE-NQO1 transcriptional pathway," Atherosclerosis, vol. 211, no. 1, pp. 69-76, 2010.

[82] R. Zhao, Q.-W. Li, J. Li, and T. Zhang, "Protective effect of Lycium barbarum polysaccharide 4 on kidneys in streptozotocin-induced diabetic rats," Canadian Journal of Physiology and Pharmacology, vol. 87, no. 9, pp. 711-719, 2009. 
[83] Q. Wen, T. Liang, F. Qin et al., "Lyoniresinol $3 \alpha-\mathrm{O}-\beta-\mathrm{D}-$ glucopyranoside-mediated hypoglycaemia and its influence on apoptosis-regulatory protein expression in the injured kidneys of streptozotocin-induced mice," PLoS ONE, vol. 8, no. 12, Article ID e81772, 2013.

[84] B. Wang, Q. Ni, X. Wang, and L. Lin, "Meta-analysis of the clinical effect of ligustrazine on diabetic nephropathy," The American Journal of Chinese Medicine, vol. 40, no. 1, pp. 25-37, 2012.

[85] M. Zhang, L. Feng, J. Gu et al., "The attenuation of Moutan cortex on oxidative stress for renal injury in AGEs-induced mesangial cell dysfunction and streptozotocin-induced diabetic nephropathy rats," Oxidative Medicine and Cellular Longevity, vol. 2014, Article ID 463815, 13 pages, 2014.

[86] M.-H. Zhang, L. Feng, J.-F. Gu, J. Jiang, and X.-B. Jia, “Effect of Moutan Cortex on AGEs-induced mesangial cell proliferation and basement membrane thickening," Zhongguo zhongyao zazhi, vol. 39, no. 3, pp. 478-482, 2014.

[87] M.-H. Zhang, L. Feng, M.-M. Zhu et al., "The anti-inflammation effect of Moutan Cortex on advanced glycation end products-induced rat mesangial cells dysfunction and Highglucose-fat diet and streptozotocin-induced diabetic nephropathy rats," Journal of Ethnopharmacology, vol. 151, no. 1, pp. 591600, 2014.

[88] T. Yokozawa, N. Yamabe, H. Y. Kim et al., "Protective effects of morroniside isolated from corni fructus against renal damage in streptozotocin-induced diabetic rats," Biological \& Pharmaceutical Bulletin, vol. 31, no. 7, pp. 1422-1428, 2008.

[89] Q.-N. Tu, H. Dong, and F.-E. Lu, "Effects of panax notoginoside on the nephropathy in rats with type 1 diabetes mellitus," Chinese Journal of Integrative Medicine, vol. 17, no. 8, pp. 612615, 2011.

[90] Q. Tu, J. Qin, H. Dong, F. Lu, and W. Guan, "Effects of Panax notoginoside on the expression of TGF-betal and Smad-7 in renal tissues of diabetic rats," Journal of Huazhong University of Science and Technology-Medical Science, vol. 31, no. 2, pp. 190193, 2011.

[91] Q.-X. Li, H.-J. Zhong, H.-R. Gong et al., "Effect of compound Puerarin on the collage IV in streptozotocin-induced diabetic nephropathy rats," Chinese Journal of Preventive Medicine, vol. 42, no. 4, pp. 254-259, 2008.

[92] Y. Zhong, X. Zhang, X. Cai, K. Wang, Y. Chen, and Y. Deng, "Puerarin attenuated early diabetic kidney injury through down-regulation of matrix metalloproteinase 9 in streptozotocin-induced diabetic rats," PLoS ONE, vol. 9, no. 1, Article ID e85690, 2014.

[93] Y. Zhang, H. Wang, L. Yu, and J. Chen, “The Puerarin improves renal function in STZ-induced diabetic rats by attenuating eNOS expression," Renal Failure, vol. 37, no. 4, pp. 699-703, 2015.

[94] Q. Gao, W.-S. Qin, Z.-H. Jia et al., "Rhein improves renal lesion and ameliorates dyslipidemia in $\mathrm{db} / \mathrm{db}$ mice with diabetic nephropathy," Planta Medica, vol. 76, no. 1, pp. 27-33, 2010.

[95] D.-Q. Yu, Y. Gao, and X.-H. Liu, "Effects of Rhein on the hypertrophy of renal proximal tubular epithelial cells induced by high glucose and angiotensin II in rats," Journal of Chinese Medicinal Materials, vol. 33, no. 4, pp. 570-574, 2010.

[96] Z.-S. Wang, F. Gao, and F.-E. Lu, "Effect of ethanol extract of Rhodiola rosea on the early nephropathy in type 2 diabetic rats," Journal of Huazhong University of Science and TechnologyMedical Science, vol. 33, no. 3, pp. 375-378, 2013.
[97] Y. Zhou, Q. Liao, Y. Luo, Z. Qing, Q. Zhang, and G. He, “Renal protective effect of Rosa laevigata Michx. by the inhibition of oxidative stress in streptozotocin-induced diabetic rats," Molecular Medicine Reports, vol. 5, no. 6, pp. 1548-1554, 2012.

[98] X.-W. Li, Y. Liu, W. Hao, and J.-R. Yang, "Sequoyitol ameliorates diabetic nephropathy in diabetic rats induced with a high-fat diet and a low dose of streptozotocin," Canadian Journal of Physiology and Pharmacology, vol. 92, no. 5, pp. 405-417, 2014.

[99] H.-W. Chen, S.-L. Wang, and X.-Y. Chen, "Preliminary study on effects of sodium ferulate in treating diabetic nephropathy," Zhongguo Zhongxiyi Jiehe Zazhi, vol. 26, no. 9, pp. 803-806, 2006.

[100] S. Zhang, J. Yang, H. Li et al., "Skimmin, a coumarin, suppresses the streptozotocin-induced diabetic nephropathy in wistar rats," European Journal of Pharmacology, vol. 692, no. 1-3, pp. 78-83, 2012.

[101] G. Liu, G.-J. Guan, T.-G. Qi et al., "Protective effects of Salvia miltiorrhiza on rats with streptozotocin diabetes and its mechanism," Journal of Chinese Integrative Medicine, vol. 3, no. 6, pp. 459-462, 2005.

[102] S.-H. Lee, Y.-S. Kim, S.-J. Lee, and B.-C. Lee, “The protective effect of Salvia miltiorrhiza in an animal model of early experimentally induced diabetic nephropathy," Journal of Ethnopharmacology, vol. 137, no. 3, pp. 1409-1414, 2011.

[103] D. Yin, J. Yin, Y. Yang, S. Chen, and X. Gao, "Renoprotection of Danshen Injection on streptozotocin-induced diabetic rats, associated with tubular function and structure," Journal of Ethnopharmacology, vol. 151, no. 1, pp. 667-674, 2014.

[104] J. Su, P. Zhang, J.-J. Zhang, X.-M. Qi, Y.-G. Wu, and J.-J. Shen, "Effects of total glucosides of paeony on oxidative stress in the kidney from diabetic rats," Phytomedicine, vol. 17, no. 3-4, pp. 254-260, 2010.

[105] B. C. Chang, W. D. Chen, Y. Zhang, P. Yang, L. Liu, and J. Wang, "Effect of total glucosides of paeony on Wnt/beta-catenin signal transduction pathway expression in kidney of diabetic rats," Zhongguo Zhongyao Zazhi, vol. 39, no. 19, pp. 3829-3835, 2014.

[106] Q.-H. Yang, Y. Liang, Q. Xu, Y. Zhang, L. Xiao, and L.-Y. Si, "Protective effect of tetramethylpyrazine isolated from Ligusticum chuanxiong on nephropathy in rats with streptozotocininduced diabetes," Phytomedicine, vol. 18, no. 13, pp. 1148-1152, 2011.

[107] Q. Gao, W. Shen, W. Qin et al., “Treatment of db/db diabetic mice with triptolide: a novel therapy for diabetic nephropathy," Nephrology Dialysis Transplantation, vol. 25, no. 11, pp. 35393547, 2010.

[108] R.-X. Ma, N. Zhao, and W. Zhang, "The effects and mechanism of Tripterygium wilfordii Hook F combination with irbesartan on urinary podocyte excretion in diabetic nephropathy patients," Zhonghua Nei Ke Za Zhi, vol. 52, no. 6, pp. 469-473, 2013.

[109] X.-P. Sun, N.-S. Wang, Q. Xue, and F. Wang, "Volatile oil of Magnolia biondii inhibits expressions of P-selectin protein in serum and renal tissue of rats with diabetic nephropathy," Journal of Chinese Integrative Medicine, vol. 6, no. 5, pp. 524$529,2008$.

[110] H.-W. Gu, Z.-H. Ni, L.-Y. Gu et al., "Effects of Astragalus on expression of renal angiopoietin receptor Tie-2 in diabetic rats," Journal of Chinese Integrative Medicine, vol. 5, no. 5, pp. 536540, 2007.

[111] D. Tang, B. He, Z.-G. Zheng et al., "Inhibitory effects of two major isoflavonoids in Radix Astragali on high glucose-induced 
mesangial cells proliferation and AGEs-induced endothelial cells apoptosis," Planta Medica, vol. 77, no. 7, pp. 729-732, 2011.

[112] K. Agyemang, L. Han, E. Liu, Y. Zhang, T. Wang, and X. Gao, "Recent advances in Astragalus membranaceus antidiabetic research: pharmacological effects of its phytochemical constituents," Evidence-Based Complementary and Alternative Medicine, vol. 2013, Article ID 654643, 9 pages, 2013.

[113] Q. Wang, X. Shao, W. Xu et al., "Astragalosides IV inhibits high glucose-induced cell apoptosis through HGF activation in cultured human tubular epithelial cells," Renal Failure, vol. 36, no. 3, pp. 400-406, 2014.

[114] W. Wu, H. Geng, Z. Liu, H. Li, and Z. Zhu, "Effect of curcumin on rats/mice with diabetic nephropathy: a systematic review and meta-analysis of randomized controlled trials," Journal of Traditional Chinese Medicine, vol. 34, no. 4, pp. 419-429, 2014.

[115] Q. Sun, G. Yang, M. Zhang, M. Zhang, S. Chen, and P. Chen, "Effect of Huangshukuihua (Flos Abelmoschi Manihot) on diabetic nephropathy: a meta-analysis," Journal of Traditional Chinese Medicine, vol. 35, no. 1, pp. 15-20, 2015.

[116] N. Yamabe, K. S. Kang, E. Goto, T. Tanaka, and T. Yokozawa, "Beneficial effect of Corni Fructus, a constituent of Hachimijio-gan, on advanced glycation end-product-mediated renal injury in streptozotocin-treated diabetic rats," Biological \& Pharmaceutical Bulletin, vol. 30, no. 3, pp. 520-526, 2007.

[117] W. Ma, K.-J. Wang, C.-S. Cheng et al., "Bioactive compounds from Cornus officinalis fruits and their effects on diabetic nephropathy," Journal of Ethnopharmacology, vol. 153, no. 3, pp. 840-845, 2014.

[118] X. Ma, X. Xie, C. Zuo, and J. Fan, "Effects of ginsenoside Rg1 on streptozocin-induced diabetic nephropathy in rats," Journal of Biomedical Engineering, vol. 27, no. 2, pp. 342-347, 2010.

[119] N. Mao, Y. Cheng, X.-L. Shi et al., "Ginsenoside Rg1 protects mouse podocytes from aldosterone-induced injury in vitro," Acta Pharmacologica Sinica, vol. 35, no. 4, pp. 513-522, 2014.

[120] T. Yokozawa, H. Y. Kim, and N. Yamabe, "Amelioration of diabetic nephropathy by dried Rehmanniae Radix (Di Huang) extract," The American Journal of Chinese Medicine, vol. 32, no. 6, pp. 829-839, 2004.

[121] Z. Dong and C. X. Chen, "Effect of catalpol on diabetic nephropathy in rats," Phytomedicine, vol. 20, no. 11, pp. 10231029, 2013.

[122] H. Yin, X. Yan, and K. H. Yang, "Systematic review on sodium ferulate for treatment of diabetic nephropathy," Zhongguo Zhongxiyi Jiehe Zazhi, vol. 29, no. 11, pp. 970-974, 2009.

[123] H. Zhang, W. Sun, Y. Wan et al., "Preventive effects of multi-glycoside of Tripterygium wilfordii on glomerular lesions in experimental diabetic nephropathy," Zhongguo Zhongyao Zazhi, vol. 35, no. 11, pp. 1460-1465, 2010.

[124] J.-P. Li, X.-L. He, and Q. Li, "Clinical study on treatment of early diabetic nephropathy by tangshenling combined with telmisartan," Chinese Journal of Integrated Traditional and Western Medicine, vol. 26, no. 5, pp. 415-418, 2006.

[125] X.-L. He, J.-P. Li, Y.-P. Chen, Z.-G. Zhang, W.-Q. Lin, and J.H. Chen, "Effects of Tangshenling Mixture and benazepril on rats with diabetic nephropathy and its mechanism," Journal of Chinese Integrative Medicine, vol. 4, no. 1, pp. 43-47, 2006.

[126] H.-X. Song, J. Gong, and W. Chen, "Effect of triptolide on urinary monocyte chemottractant protein-1 in patients with diabetic nephropathy," Chinese Journal of Integrated Traditional and Western Medicine, vol. 25, no. 5, pp. 416-418, 2005.
[127] J. Song, Y. H. Li, and X. D. Yang, "Effect of combined therapy with bailing capsule and benazepril on urinary albumin excretion rate and C-reactive protein in patients with early diabetic nephropathy," Chinese Journal of Integrated Traditional and Western Medicine, vol. 29, no. 9, pp. 791-793, 2009.

[128] D.-Z. Guo, Y.-H. Wang, and Z.-Q. Chen, "Effect of treatment in 39 patients with diabetic nephropathy by safflor yellow and benazepril in combination," Chinese Journal of Integrated Traditional and Western Medicine, vol. 28, no. 4, pp. 360-363, 2008.

[129] L. Zhao, L.-G. Lan, X.-L. Min et al., "Integrated treatment of traditional Chinese medicine and western medicine for early- and intermediate-stage diabetic nephropathy," Journal of Southern Medical University, vol. 27, no. 7, pp. 1052-1055, 2007. 


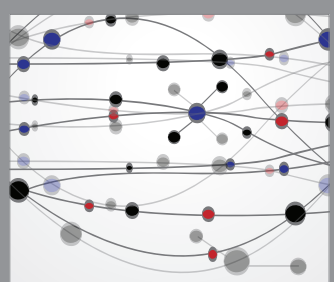

The Scientific World Journal
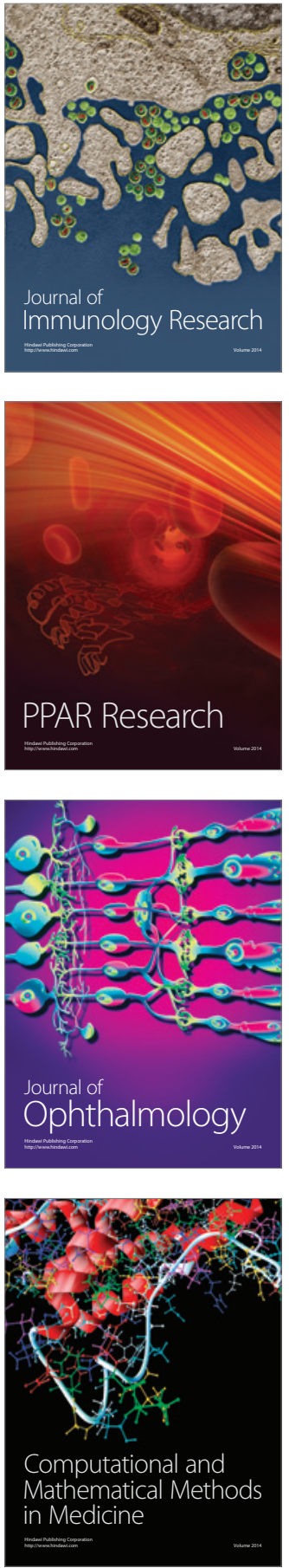

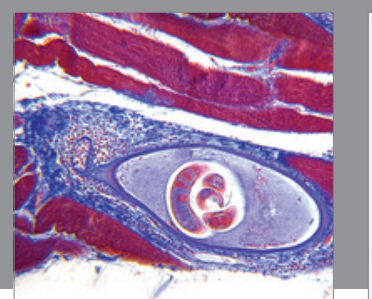

Gastroenterology Research and Practice

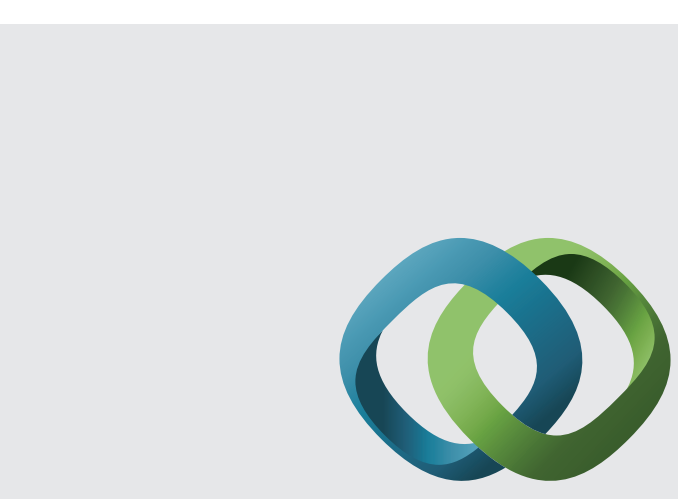

\section{Hindawi}

Submit your manuscripts at

http://www.hindawi.com
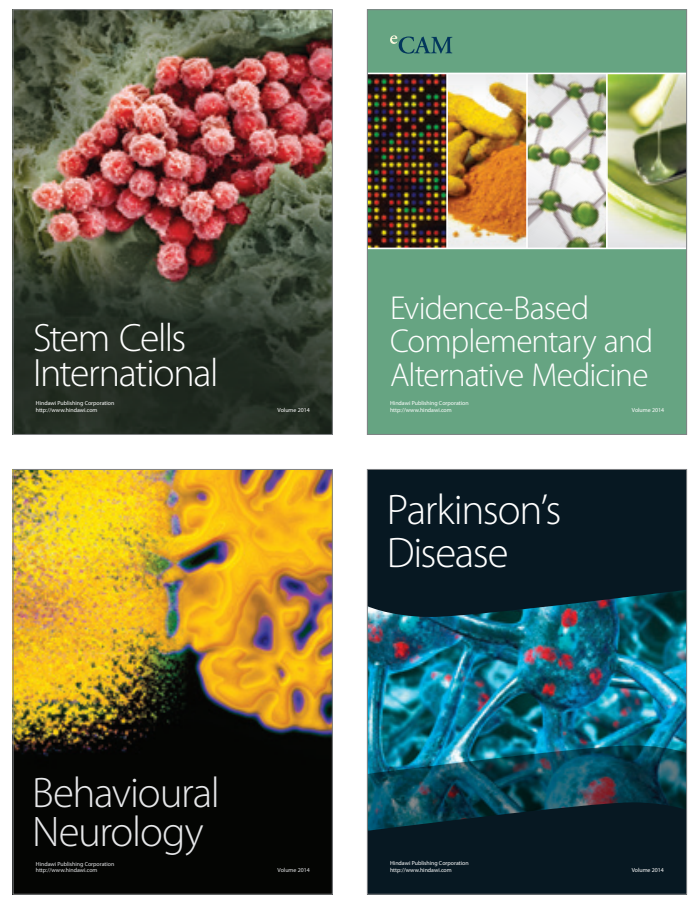
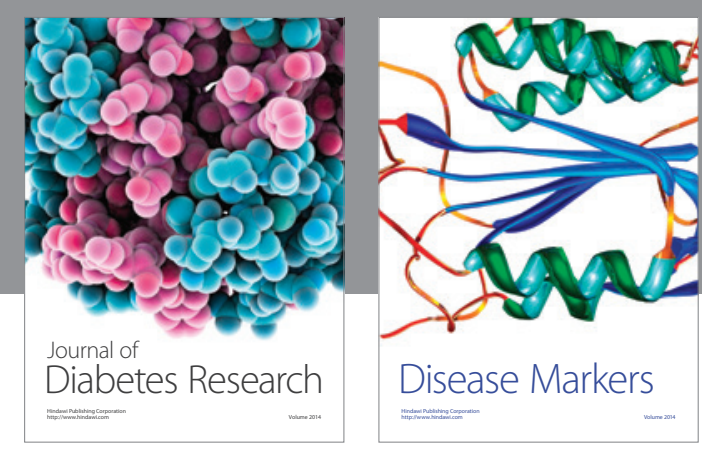

Disease Markers
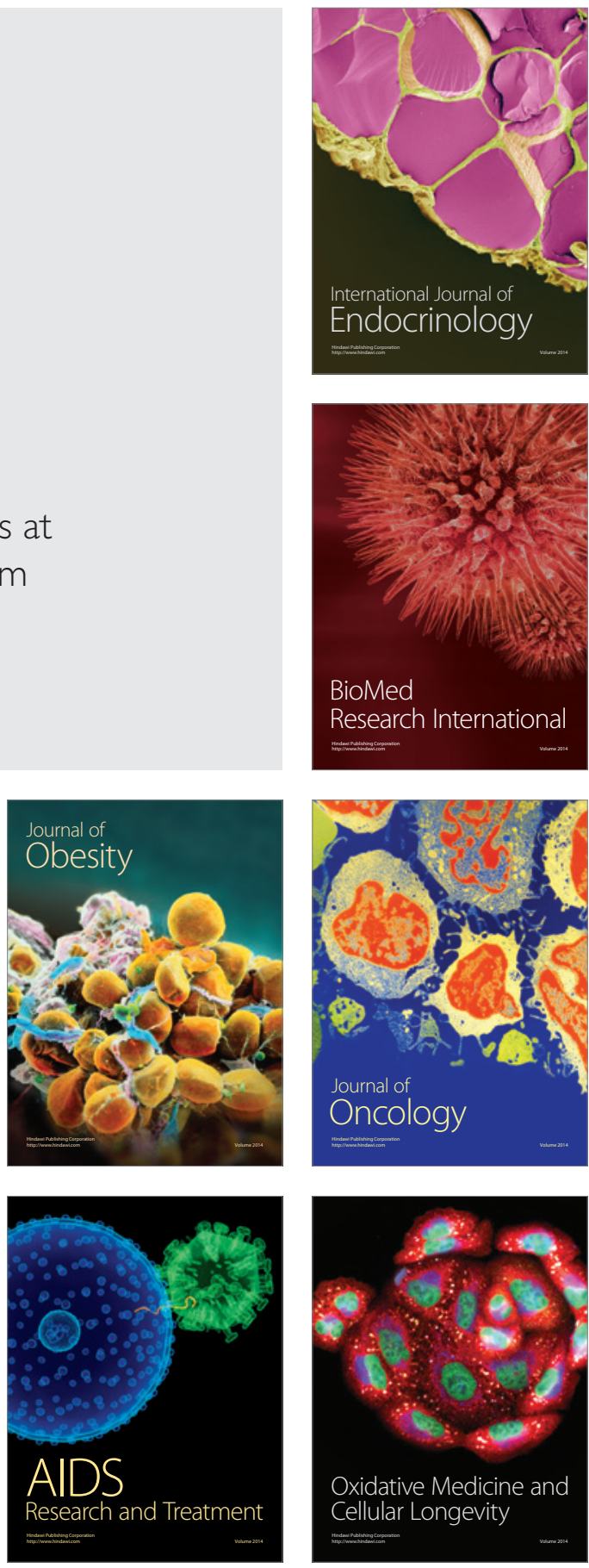